\title{
Torsion units for a Ree group, Tits group and a Steinberg triality group
}

\author{
Joe Gildea ${ }^{1}$
}

Received: 5 November 2015 / Accepted: 5 December 2015 / Published online: 28 December 2015

(C) The Author(s) 2015. This article is published with open access at Springerlink.com

\begin{abstract}
We investigate the Zassenhaus conjecture for the Steinberg triality group ${ }^{3} D_{4}\left(2^{3}\right)$, Tits group ${ }^{2} F_{4}(2)^{\prime}$ and the Ree group ${ }^{2} F_{4}(2)$. Consequently, we prove that the Prime Graph question is true for all three groups.
\end{abstract}

Keywords Zassenhaus conjecture · Prime graph question · Torsion unit · Partial augmentation · Integral group ring

Mathematics Subject Classification 16 S34 $\cdot 20 \mathrm{C} 05$

\section{Introduction and main results}

Let $U(\mathbb{Z} G)$ be the unit group of the integral group ring of a finite group $G$. It is well known that

$$
U(\mathbb{Z} G)=\{ \pm 1\} \times V(\mathbb{Z} G),
$$

where $V(\mathbb{Z} G)$ is the group of units of augmentation one. Throughout this paper, $G$ will always represent a finite group and torsion units will always represent torsion units in $V(\mathbb{Z} G) \backslash\{1\}$. The next conjecture is very important in theory of integral group rings:

Conjecture 1 If $G$ is a finite group, then for each torsion unit $u \in V(\mathbb{Z} G)$ there exists $g \in G$, such that $|u|=|g|$.

Hans Zassenhaus formulated a stronger version of this conjecture in [1], which states:

Conjecture 2 A torsion unit in $V(\mathbb{Z} G)$ is said to be rationally conjugate to a group element if it is conjugate to an element of $G$ by a unit of the rational group ring $\mathbb{Q} G$.

Joe Gildea

j.gildea@chester.ac.uk

1 Department of Mathematics, Faculty of Science and Engineering, University of Chester, Chester, England 
This conjecture was confirmed for Nilpotent Groups in [2,3] and cyclic-by-abelian groups in [4]. However, these techniques do not transfer to simple groups. The Luthar-Passi Method (which was introduced in [5]) is the main investigative tool for simple groups in relation to the Zassenhaus conjecture. The Zassenhaus conjecture was confirmed true for all groups up to order 71 in [6]. This conjecture was also validated for $A_{5}, S_{5}$, central extensions of $S_{5}$ and other simple finite groups in [5,7-9]. In [10] partial results were given for $A_{6}$, and the remaining cases were dealt with in [11]. Alternating groups of higher order were also considered in $[12,13]$. Additionally, it was proved for $\operatorname{PSL}(2, p)$ when $p=\{7,11,13\}$ in [14] and $p=\{8,17\}$ in [15] and $\operatorname{PSL}(2, p)$ when $p=\{19,23\}$ in [16]. See [17,18] for further results regarding $P S L(2, p)$.

Let $H$ be a group with a torsion part $t(H)$ of finite exponent and $\# H$ be the set of primes dividing the order of elements from the set $t(H)$. The prime graph of $H$ (denoted by $\pi(H)$ ) is a graph with vertices labeled by primes from $\# H$, such that vertices $p$ and $q$ are adjacent if and only if there is an element of order $p q$ in the group $H$. The following, was composed as a problem in [19] (Problem 37):

Question 1 (Prime Graph Question) If $G$ is a finite group, then $\pi(G)=\pi(V(\mathbb{Z} G))$.

This question is upheld for Frobenius and Solvable groups in [20]. It was also confirmed for some Sporadic Simple groups in [21-32]. Additionally, it was confirmed for the sympletic simple group $S_{4}(4)$ in [33]. The prime graph question is an intermediate step towards the Zassenhaus conjecture.

The family of Steinberg Triality groups (denoted by ${ }^{3} D_{4}\left(q^{3}\right)$ ) were introduced by Steinberg in [34]. The Ree groups ${ }^{2} F_{4}\left(2^{n+1}\right)$ were initially constructed in [35]. These groups are simple if $n \geq 1$ and Tits constructed a new simple group from the derived subgroup of ${ }^{2} F_{4}(2)$ (denoted by $\left.{ }^{2} F_{4}(2)^{\prime}\right)$ in [36].

We combine the Luthar-Passi Method together with techniques developed in $[14,37]$ to obtain our results. Our main results are as follows:

Theorem 1 Let $G={ }^{3} D_{4}\left(2^{3}\right)$ and $u$ be a torsion unit of $V(\mathbb{Z} G)$. Subsequently the following conditions hold:

(i) If $|u| \in\{7\}$, then $u$ is rationally conjugate to some $g$ in $G$.

(ii) There are no elements of orders 26,39 or 91 in $V(\mathbb{Z} G)$.

(iii) If $|u|=2$, then $v_{r x}=0 \forall r x \notin\left\{v_{2 a}, v_{2 b}\right\}$ and

$$
\left(v_{2 a}, v_{2 b}\right) \in\{(2,-1),(1,0),(0,1),(-1,2),(-2,3)\} .
$$

(iv) If $|u|=3$, then $v_{r x}=0 \forall r x \notin\left\{v_{3 a}, v_{3 b}\right\}$ and

$$
\left(v_{3 a}, v_{3 b}\right) \in\{(3,-2),(2,-1),(1,0),(0,1),(-1,2)\} .
$$

(v) If $|u|=13$, then $v_{r x}=0 \forall r x \notin\left\{v_{13 a}, v_{13 b}, v_{13 c}\right\}$ and

$$
\left(v_{13 a}, v_{13 b}, v_{13 c}\right) \in\{(1,-1,1),(1,0,0),(0,0,1),(1,1,-1),(0,1,0),(-1,1,1)\} .
$$

Theorem 2 Let $G={ }^{2} F_{4}(2)^{\prime}$ and $u$ be a torsion unit of $V(\mathbb{Z} G)$. Subsequently the following conditions hold:

(i) If $|u| \in\{3,5\}$, then $u$ is rationally conjugate to some $g$ in $G$.

(ii) There are no elements of orders 15, 26, 39 or 65 in $V(\mathbb{Z} G)$.

(iii) If $|u|=2$, then $v_{r x}=0 \forall r x \notin\left\{v_{2 a}, v_{2 b}\right\}$ and

$$
\left(v_{2 a}, v_{2 b}\right) \in\{(3,-2),(2,-1),(1,0),(0,1),(-1,2),(-2,3),(-3,4)\} .
$$


(iv) If $|u|=10$, then $v_{r x}=0 \forall r x \notin\left\{v_{2 a}, v_{2 b}, v_{5 a}, v_{10 a}\right\}$ and

$$
\begin{aligned}
\left(v_{2 a}, v_{2 b}, v_{5 a}, v_{10 a}\right) \in\{ & (-2,-2,0,5),(-1,-3,0,5),(-1,-3,5,0),(-1,-1,0,3), \\
& (-1,-1,5,-2),(-1,1,0,1),(0,-4,5,0),(0,-2,0,3), \\
& (0,-2,5,-2),(0,0,0,1),(0,0,5,-4),(0,2,0,-1), \\
& (0,4,-5,2),(0,4,0,-3),(1,1,0,-1),(1,3,-5,2), \\
& (1,3,0,-3),(2,2,0,-3)\} .
\end{aligned}
$$

(v) If $|u|=13$, then $v_{r x}=0 \forall r x \notin\left\{v_{13 a}, v_{13 b}\right\}$ and

$$
\begin{aligned}
\left(v_{13 a}, v_{13 b}\right) \in\{ & (9,-8),(8,-7),(7,-6),(6,-5),(5,-4),(4,-3),(3,-2),(2,-1), \\
& (1,0),(0,1),(-1,2),(-2,3),(-3,4),(-4,5),(-5,6),(-6,7), \\
& (-7,8),(-8,9)\} .
\end{aligned}
$$

Theorem 3 Let $G={ }^{2} F_{4}(2)$ and $u$ be a torsion unit of $V(\mathbb{Z} G)$. Subsequently the following conditions hold:

(i) If $|u| \in\{3,5,13\}$, then $u$ is rationally conjugate to some $g$ in $G$.

(ii) There are no elements of orders $15,26,39$ or 65 in $V(\mathbb{Z} G)$.

(iii) If $|u|=2$, then $v_{r x}=0 \forall r x \notin\left\{v_{2 a}, v_{2 b}\right\}$ and

$$
\left(v_{2 a}, v_{2 b}\right) \in\{(3,-2),(2,-1),(1,0),(0,1),(-1,2),(-2,3),(-3,4)\} .
$$

(iv) If $|u|=10$, then $v_{r x}=0 \forall r x \notin\left\{v_{2 a}, v_{2 b}, v_{5 a}, v_{10 a}\right\}$ and

$$
\begin{aligned}
\left(v_{2 a}, v_{2 b}, v_{5 a}, v_{10 a}\right) \in\{ & (-2,-2,0,5),(-1,-3,0,5),(-1,-3,5,0),(-1,-1,0,3), \\
& (-1,-1,5,-2),(-1,1,0,1),(0,-4,5,0),(0,-2,0,3), \\
& (0,-2,5,-2),(0,0,0,1),(0,0,5,-4),(0,2,0,-1), \\
& (0,4,-5,2),(0,4,0,-3),(1,1,0,-1),(1,3,-5,2), \\
& (1,3,0,-3),(2,2,0,-3)\} .
\end{aligned}
$$

Corollary 1 The Prime Graph question is true for the groups ${ }^{3} D_{4}(2),{ }^{2} F_{4}(2){ }^{\prime}$ and ${ }^{2} F_{4}(2)$.

Let $u=\sum a_{g} g$ be a torsion unit of $V(\mathbb{Z} G)$. Then, the sum $\sum_{g \in X^{G}} a_{g} \in \mathbb{Z}$ is the partial augmentation (denoted by $\varepsilon_{C}(u)$ ) of $u$ with respect to it's conjugacy classes $X^{G}$ in $G$. Let $v_{i}=\varepsilon_{C_{i}}(u)$ be the $i$-th partial augmentation of $u$. It was proved that $v_{1}=0$ and $v_{j}=0$ if the conjugacy class $C_{j}$ consists of a central element by G. Higman and S. D. Berman [38]. Therefore, $v_{2}+v_{3}+\cdots+v_{l}=1$, where $l$ denotes the number of non-central conjugacy classes of $G$.

Proposition 1 ([39]) Let $u$ be torsion unit of $V(\mathbb{Z} G)$. The order of $u$ divides the exponent of G.

The following propositions provide relationships between the partial augmentations and the order of a torsion unit.

Proposition 2 (Proposition 3.1 in [37]) Let u be a torsion unit of $V(\mathbb{Z} G)$. Let $C$ be a conjugacy class of $G$. If $p$ is a prime dividing the order of a representative of $C$ but not the order of $u$ then the partial augmentation $\varepsilon_{C}(u)=0$.

Proposition 3 (Proposition 2.2 in [14]) Let $G$ be a finite group and let $u$ be a torsion unit in $V(\mathbb{Z} G)$. 
(i) If $u$ has order $p^{n}$, then $\varepsilon_{x}(u)=0$ for every $x$ of $G$ whose p-part is of order strictly greater than $p^{n}$.

(ii) If $x$ is an element of $G$ whose p-part, for some prime, has order strictly greater than the order of the p-part of $u$, then $\varepsilon_{x}(u)=0$.

Proposition 4 ([5]) Let $u$ be a torsion unit of $V(\mathbb{Z} G)$ of order $k$. Then $u$ is conjugate in $\mathbb{Q} G$ to an element $g \in G$ iff for each d dividing $k$ there is precisely one conjugacy class $v i_{d}$ with partial augmentation $\varepsilon_{v i_{d}}\left(u^{d}\right) \neq 0$.

Proposition 5 ([5]) Let $p$ be equal to zero or a prime divisor of $|G|$. Suppose that $u$ is an element of $V(\mathbb{Z} G)$ of order $k$. Let $z$ be a primitive kth root of unity. Then for every integer $l$ and any character $\chi$ of $G$, the number

$$
\mu_{l}(u, \chi, p)=\frac{1}{k} \sum_{d \mid k} \operatorname{Tr}_{\mathbb{Q}\left(z^{d}\right) / \mathbb{Q}}\left\{\chi\left(u^{d}\right) z^{-d l}\right\}
$$

is a non-negative integer.

We will use the notation $\mu_{l}(u, \chi, *)$ when $p=0$. The LAGUNA package [40] for the GAP system [41] is a very useful tool when calculating $\mu_{l}(u, \chi, p)$.

\section{Proof of Theorem 1}

Let $G={ }^{3} D_{4}\left(2^{3}\right)$. Clearly $|G|=211341312=2^{12} \cdot 3^{4} \cdot 7^{2} \cdot 13$ and $\exp (G)=6552=$ $2^{3} \cdot 3^{2} \cdot 7 \cdot 13$. Initially, for any torsion unit of $V(\mathbb{Z} G)$ of order $k$ :

$$
\begin{gathered}
v_{2 a}+v_{2 b}+v_{3 a}+v_{3 b}+v_{4 a}+v_{4 b}+v_{4 c}+v_{6 a}+v_{6 b}+v_{7 a}+v_{7 b}+v_{7 c}+v_{7 d}+v_{8 a} \\
+v_{8 b}+v_{9 a}+v_{9 b}+v_{9 c}+v_{12 a}+v_{13 a}+v_{13 b}+v_{13 c}+v_{14 a}+v_{14 b}+v_{14 c} \\
+v_{18 a}+v_{18 b}+v_{18 c}+v_{21 a}+v_{21 b}+v_{21 c}+v_{28 a}+v_{28 b}+v_{28 c}=1 .
\end{gathered}
$$

In order to prove that the Zassenhaus Conjecture holds, we need to consider torsion units of $V(\mathbb{Z} G)$ of order $2,3,4,6,7,8,9,12,13,14,18,21,28,24,26,36,39,42,56,63$ and 91 (by Proposition 1). For the purpose of this paper and due to the complexity of certain orders, we shall consider elements of order 2, 3, 7, 13, 26, 39 and 91. We shall now consider each case separately.

Case $(i)$. Let $u \in V(\mathbb{Z} G)$ where $|u|=2$. Using Propositions 2 and 3,

$$
v_{2 a}+v_{2 b}=1 \text {. }
$$

Applying Proposition 5, we obtain the following system of inequalities:

$$
\begin{aligned}
& \mu_{0}\left(u, \chi_{2}, *\right)=\frac{1}{2}\left(-2 \gamma_{1}+26\right) \geq 0 ; \quad \mu_{1}\left(u, \chi_{2}, *\right)=\frac{1}{2}\left(2 \gamma_{1}+26\right) \geq 0 ; \\
& \mu_{0}\left(u, \chi_{3}, *\right)=\frac{1}{2}\left(4 \gamma_{2}+52\right) \geq 0 ; \quad \mu_{1}\left(u, \chi_{3}, *\right)=\frac{1}{2}\left(-4 \gamma_{2}+52\right) \geq 0
\end{aligned}
$$

where $\gamma_{1}=3 v_{2 a}-v_{2 b}$ and $\gamma_{2}=5 v_{2 a}-v_{2 b}$. It follows that the only possible integer solutions for $\left(v_{2 a}, v_{2 b}\right)$ are listed in part (iii) of Theorem 1.

Case $(i i)$. Let $u \in V(\mathbb{Z} G)$ where $|u|=3$. Using Propositions 2 and 3,

$$
v_{3 a}+v_{3 b}=1 \text {. }
$$


Applying Proposition 5, we obtain the following system of inequalities:

$$
\begin{aligned}
& \mu_{0}\left(u, \chi_{3}, *\right)=\frac{1}{3}\left(2 \gamma_{1}+52\right) \geq 0 ; \quad \mu_{1}\left(u, \chi_{3}, *\right)=\frac{1}{3}\left(-\gamma_{1}+52\right) \geq 0 ; \\
& \mu_{0}\left(u, \chi_{2}, 2\right)=\frac{1}{3}\left(2 \gamma_{2}+8\right) \geq 0 ; \quad \mu_{1}\left(u, \chi_{2}, 2\right)=\frac{1}{3}\left(-\gamma_{2}+8\right) \geq 0
\end{aligned}
$$

where $\gamma_{1}=7 v_{3 a}-2 v_{3 b}$ and $\gamma_{2}=2 v_{3 a}-v_{3 b}$. It follows that the only possible integer solutions for $\left(v_{2 a}, v_{2 b}\right)$ are listed in part $(i v)$ of Theorem 1.

Case (iii). Let $u \in V(\mathbb{Z} G)$ where $|u|=7$. Using Propositions 2 and 3 ,

$$
v_{7 a}+v_{7 b}+v_{7 c}+v_{7 d}=1 .
$$

Applying Proposition 5, we obtain:

$$
\begin{aligned}
& \mu_{0}\left(u, \chi_{2}, *\right)=\frac{1}{7}\left(6 \gamma_{1}+26\right) \geq 0 ; \quad \mu_{1}\left(u, \chi_{2}, *\right)=\frac{1}{7}\left(-\gamma_{1}+26\right) \geq 0 ; \\
& \mu_{0}\left(u, \chi_{5}, *\right)=\frac{1}{7}\left(42 \gamma_{2}+273\right) \geq 0 ; \quad \mu_{1}\left(u, \chi_{5}, *\right)=\frac{1}{7}\left(-7 \gamma_{2}+273\right) \geq 0 ; \\
& \mu_{0}\left(u, \chi_{6}, *\right)=\frac{1}{7}\left(6 \gamma_{3}+324\right) \geq 0 ; \quad \mu_{1}\left(u, \chi_{6}, *\right)=\frac{1}{7}\left(-\gamma_{3}+324\right) \geq 0 ; \\
& \mu_{0}\left(u, \chi_{10}, *\right)=\frac{1}{7}\left(6 \gamma_{4}+468\right) \geq 0 ; \quad \mu_{1}\left(u, \chi_{10}, *\right)=\frac{1}{7}\left(-\gamma_{4}+468\right) \geq 0 \text {; } \\
& \mu_{0}\left(u, \chi_{6}, 13\right)=\frac{1}{7}\left(6 \gamma_{5}+323\right) \geq 0 ; \quad \mu_{1}\left(u, \chi_{6}, 13\right)=\frac{1}{7}\left(-\gamma_{5}+323\right) \geq 0 \text {; } \\
& \mu_{0}\left(u, \chi_{2}, 3\right)=\frac{1}{7}\left(4 \gamma_{6}+25\right) \geq 0 ; \quad \mu_{1}\left(u, \chi_{2}, 3\right)=\frac{1}{7}\left(-\gamma_{6}+25\right) \geq 0 \text {; } \\
& \mu_{0}\left(u, \chi_{2}, 2\right)=\frac{1}{7}\left(-2 \gamma_{6}+8\right) \geq 0 ; \quad \mu_{0}\left(u, \chi_{12}, *\right)=\frac{1}{7}\left(6 \gamma_{7}+1053\right) \geq 0 ; \\
& \mu_{1}\left(u, \chi_{12}, *\right)=\frac{1}{7}\left(-\gamma_{7}+1053\right) \geq 0 ; \quad \mu_{1}\left(u, \chi_{2}, 2\right)=\frac{1}{7}\left(\gamma_{8}+8\right) \geq 0 \text {; } \\
& \mu_{3}\left(u, \chi_{2}, 2\right)=\frac{1}{7}\left(\gamma_{9}+8\right) \geq 0 ; \quad \mu_{2}\left(u, \chi_{2}, 2\right)=\frac{1}{7}\left(\gamma_{10}+8\right) \geq 0 ; \\
& \mu_{1}\left(u, \chi_{18}, *\right)=\frac{1}{7}\left(\gamma_{11}+2106\right) \geq 0 ; \quad \mu_{2}\left(u, \chi_{18}, *\right)=\frac{1}{7}\left(\gamma_{12}+2106\right) \geq 0 ; \\
& \mu_{3}\left(u, \chi_{18}, *\right)=\frac{1}{7}\left(\gamma_{13}+2106\right) \geq 0 ; \quad \mu_{0}\left(u, \chi_{25}, *\right)=\frac{1}{7}\left(\gamma_{14}+2808\right) \geq 0 \text {; } \\
& \mu_{1}\left(u, \chi_{25}, *\right)=\frac{1}{7}\left(\gamma_{15}+2808\right) \geq 0 ; \quad \mu_{2}\left(u, \chi_{25}, *\right)=\frac{1}{7}\left(\gamma_{16}+2808\right) \geq 0 \text {; } \\
& \mu_{3}\left(u, \chi_{25}, *\right)=\frac{1}{7}\left(\gamma_{17}+2808\right) \geq 0 ; \quad \mu_{1}\left(u, \chi_{6}, 2\right)=\frac{1}{7}\left(\gamma_{18}+48\right) \geq 0 \text {; } \\
& \mu_{2}\left(u, \chi_{6}, 2\right)=\frac{1}{7}\left(\gamma_{19}+48\right) \geq 0 ; \quad \mu_{3}\left(u, \chi_{6}, 2\right)=\frac{1}{7}\left(\gamma_{20}+48\right) \geq 0 ; \\
& \mu_{0}\left(u, \chi_{9}, 2\right)=\frac{1}{7}\left(\gamma_{21}+160\right) \geq 0 ; \quad \mu_{1}\left(u, \chi_{9}, 2\right)=\frac{1}{7}\left(\gamma_{22}+160\right) \geq 0 ; \\
& \mu_{2}\left(u, \chi_{9}, 2\right)=\frac{1}{7}\left(\gamma_{23}+160\right) \geq 0 ; \quad \mu_{3}\left(u, \chi_{9}, 2\right)=\frac{1}{7}\left(\gamma_{23}+160\right) \geq 0 ; \\
& \mu_{1}\left(u, \chi_{13}, 2\right)=\frac{1}{7}\left(\gamma_{25}+784\right) \geq 0 ; \quad \mu_{2}\left(u, \chi_{13}, 2\right)=\frac{1}{7}\left(\gamma_{26}+784\right) \geq 0 ; \\
& \mu_{3}\left(u, \chi_{13}, 2\right)=\frac{1}{7}\left(\gamma_{27}+784\right) \geq 0 ; \quad \mu_{1}\left(u, \chi_{22}, *\right)=\frac{1}{7}\left(\gamma_{28}+2457\right) \geq 0 ; \\
& \mu_{2}\left(u, \chi_{22}, *\right)=\frac{1}{7}\left(\gamma_{29}+2457\right) \geq 0 ; \quad \mu_{3}\left(u, \chi_{22}, *\right)=\frac{1}{7}\left(\gamma_{30}+2457\right) \geq 0
\end{aligned}
$$

where $\gamma_{1}=5 v_{7 a}+5 v_{7 b}+5 v_{7 c}-2 v_{7 d}, \gamma_{2}=v_{7 a}+v_{7 b}+v_{7 c}, \gamma_{3}=9 v_{7 a}+9 v_{7 b}+$ $9 v_{7 c}+2 v_{7 d}, \gamma_{4}=6 v_{7 a}+6 v_{7 b}+6 v_{7 c}-v_{7 d}, \gamma_{5}=8 v_{7 a}+8 v_{7 b}+8 v_{7 c}+v_{7 d}, \gamma_{6}=$ $4 v_{7 a}+4 v_{7 b}+4 v_{7 c}-3 v_{7 d}, \gamma_{7}=3 v_{7 a}+3 v_{7 b}+3 v_{7 c}-4 v_{7 d}, \gamma_{8}=-8 v_{7 a}-v_{7 b}+$ $13 v_{7 c}-v_{7 d}, \gamma_{9}=-v_{7 a}+13 v_{7 b}-8 v_{7 c}-v_{7 d}, \gamma_{10}=13 v_{7 a}-8 v_{7 b}-v_{7 c}-v_{7 d}, \gamma_{11}=$ $36 v_{7 a}-27 v_{7 b}-6 v_{7 c}+v_{7 d}, \gamma_{12}=-6 v_{7 a}+36 v_{7 b}-27 v_{7 c}+v_{7 d}, \gamma_{13}=-27 v_{7 a}-6 v_{7 b}+$ $36 v_{7 c}+v_{7 d}, \gamma_{14}=-22 v_{7 a}-22 v_{7 b}-22 v_{7 c}+6 v_{7 d}, \gamma_{15}=34 v_{7 a}-v_{7 b}-22 v_{7 c}-v_{7 d}, \gamma_{16}=$ $-22 v_{7 a}+34 v_{7 b}-v_{7 c}-v_{7 d}, \gamma_{17}=-v_{7 a}-22 v_{7 b}+34 v_{7 c}-v_{7 d}, \gamma_{18}=-20 v_{7 a}+8 v_{7 b}+$ $15 v_{7 c}+v_{7 d}, \gamma_{19}=15 v_{7 a}-20 v_{7 b}+8 v_{7 c}+v_{7 d}, \gamma_{20}=8 v_{7 a}+15 v_{7 b}-20 v_{7 c}+v_{7 d}, \gamma_{21}=$ $-34 v_{7 a}-34 v_{7 b}-34 v_{7 c}-6 v_{7 d}, \gamma_{22}=-20 v_{7 a}-13 v_{7 b}+50 v_{7 c}+v_{7 d}, \gamma_{23}=50 v_{7 a}-20 v_{7 b}-$ $13 v_{7 c}+v_{7 d}, \gamma_{24}=-13 v_{7 a}+50 v_{7 b}-20 v_{7 c}+v_{7 d}, \gamma_{25}=-35 v_{7 a}+28 v_{7 b}+7 v_{7 c}, \gamma_{26}=$ 
$7 v_{7 a}-35 v_{7 b}+28 v_{7 c}, \gamma_{27}=28 v_{7 a}+7 v_{7 b}-35 v_{7 c}, \gamma_{28}=35 v_{7 a}-14 v_{7 b}-14 v_{7 c}, \gamma_{29}=$ $-14 v_{7 a}+35 v_{7 b}-14 v_{7 c}$ and $\gamma_{30}=-14 v_{7 a}-14 v_{7 b}+35 v_{7 c}$.

It follows that the only possible integer solutions for $\left(v_{7 a}, v_{7 b}, v_{7 c}, v_{7 d}\right)$ are $(1,0,0,0)$, $(0,0,0,1),(0,0,1,0)$ and $(0,1,0,0)$. Therefore, $u$ is rationally conjugated to some element $g \in G$ by Proposition 4 .

Case $(i v)$. Let $u \in V(\mathbb{Z} G)$ where $|u|=13$. Using Propositions 2 and 3,

$$
v_{13 a}+v_{13 b}+v_{13 c}=1 \text {. }
$$

Applying Proposition 5, we obtain:

$$
\begin{gathered}
\mu_{1}\left(u, \chi_{9}, 2\right)=\frac{1}{13}\left(\gamma_{1}+160\right) \geq 0 ; \quad \mu_{1}\left(u, \chi_{6}, 2\right)=\frac{1}{13}\left(-\gamma_{1}+48\right) \geq 0 ; \\
\mu_{2}\left(u, \chi_{6}, 2\right)=\frac{1}{13}\left(\gamma_{2}+48\right) \geq 0 ; \quad \mu_{2}\left(u, \chi_{9}, 2\right)=\frac{1}{13}\left(-\gamma_{2}+160\right) \geq 0 ; \\
\mu_{1}\left(u, \chi_{2}, 2\right)=\frac{1}{13}\left(\gamma_{3}+8\right) \geq 0 ; \quad \mu_{2}\left(u, \chi_{2}, 2\right)=\frac{1}{13}\left(\gamma_{3}+8\right) \geq 0 ; \\
\mu_{4}\left(u, \chi_{2}, 2\right)=\frac{1}{13}\left(\gamma_{4}+8\right) \geq 0
\end{gathered}
$$

where $\gamma_{1}=9 v_{13 a}-4 v_{13 b}-4 v_{13 c}, \gamma_{2}=4 v_{13 a}-9 v_{13 b}+4 v_{13 c}, \gamma_{3}=5 v_{13 a}-8 v_{13 b}+5 v_{13 c}$, $\gamma_{4}=5 v_{13 a}+5 v_{13 b}-8 v_{13 c}$ and $\gamma_{5}=-8 v_{13 a}+5 v_{13 b}+5 v_{13 c}$. Clearly $\gamma_{1} \in\{9+13 k \mid$ $-13 \leq k \leq 3\}$ and $\gamma_{2} \in\{4+13 k \mid-4 \leq k \leq 12\}$. It follows that the only possible integer solutions for $\left(v_{13 a}, v_{13 b}, v_{13 c}\right)$ are listed in part $(v)$ of Theorem 1.

Case $(v)$. Let $u \in V(\mathbb{Z} G)$ where $|u|=26$. Using Propositions 2 and 3,

$$
v_{2 a}+v_{2 b}+v_{13 a}+v_{13 b}+v_{13 c}=1 \text {. }
$$

Let $\gamma_{1}=3 v_{2 a}-v_{2 b}, \gamma_{2}=5 v_{2 a}-v_{2 b}, \gamma_{3}=-28 v_{2 a}-4 v_{2 b}+v_{13 a}+v_{13 b}+v_{13 c}$, $\gamma_{4}=63 v_{2 a}+15 v_{2 b}-4 v_{13 a}-4 v_{13 b}+9 v_{13 c}$ and $\gamma_{5}=63 v_{2 a}+15 v_{2 b}+9 v_{13 a}-4 v_{13 b}-4 v_{13 c}$. We shall now separately consider the following cases involving $\chi\left(u^{n}\right)$ for $n \in\{2,13\}$ :

- $\chi\left(u^{13}\right)=m_{1} \chi(2 a)+m_{2} \chi(2 b)$ and $\chi\left(u^{2}\right)=m_{3} \chi(13 a)+m_{4} \chi(13 b)+m_{5} \chi(13 c)$ where

$$
\begin{aligned}
\left(m_{1}, m_{2}, m_{3}, m_{4}, m_{5}\right) \in\{ & (1,0,1,0,0),(1,0,0,1,0),(1,0,0,0,1),(1,0,1,-1,1), \\
& (1,0,1,1,-1),(1,0,-1,1,1),(2,-1,1,0,0), \\
& (2,-1,0,1,0),(2,-1,0,0,1),(2,-1,1,-1,1), \\
& (2,-1,1,1,-1),(2,-1,-1,1,1),(-1,2,1,0,0), \\
& (-1,2,0,1,0),(-1,2,0,0,1),(-1,2,1,-1,1), \\
& (-1,2,1,1,-1),(-1,2,-1,1,1),(-2,3,1,0,0), \\
& (-2,3,0,1,0),(-2,3,0,0,1),(-2,3,1,-1,1), \\
& (-2,3,1,1,-1),(-2,3,-1,1,1)\} .
\end{aligned}
$$

Applying Proposition 5, we obtain:

$$
\mu_{13}\left(u, \chi_{2}, *\right)=\frac{1}{26}\left(24 \gamma_{1}+8\right) \geq 0 ; \quad \mu_{0}\left(u, \chi_{2}, *\right)=\frac{1}{26}\left(-24 \gamma_{1}+44\right) \geq 0 .
$$

It follows that there are no possible integer solutions for $\left(v_{2 a}, v_{2 b}, v_{13 a}, v_{13 b}, v_{13 c}\right)$ in all cases.

- $\chi\left(u^{13}\right)=m_{1} \chi(2 a)+m_{2} \chi(2 b)$ and $\chi\left(u^{2}\right)=m_{3} \chi(13 a)+m_{4} \chi(13 b)+m_{5} \chi(13 c)$ where

$$
\left(m_{1}, m_{2}, m_{3}, m_{4}, m_{5}\right) \in\{(0,1,1,0,0),(0,1,0,1,0),(0,1,0,0,1),
$$

$$
(0,1,1,-1,1),(0,1,1,1,-1),(0,1,-1,1,1)\} \text {. }
$$


Applying Proposition 5, we obtain:

$$
\begin{gathered}
\mu_{13}\left(u, \chi_{2}, *\right)=\frac{1}{26}\left(24 \gamma_{1}+24\right) \geq 0 ; \quad \mu_{0}\left(u, \chi_{2}, *\right)=\frac{1}{26}\left(-24 \gamma_{1}+28\right) \geq 0 ; \\
\mu_{0}\left(u, \chi_{3}, *\right)=\frac{1}{26}\left(48 \gamma_{2}+48\right) \geq 0 ; \quad \mu_{13}\left(u, \chi_{3}, *\right)=\frac{1}{26}\left(-48 \gamma_{2}+56\right) \geq 0 ; \\
\mu_{1}\left(u, \chi_{4}, *\right)=\frac{1}{26}\left(\gamma_{3}+199\right) \geq 0 ; \quad \mu_{8}\left(u, \chi_{31}, *\right)=\frac{1}{26}\left(\gamma_{4}+k_{1}\right) \geq 0 ; \\
\mu_{1}\left(u, \chi_{31}, *\right)=\frac{1}{26}\left(-\gamma_{4}+k_{2}\right) \geq 0 ; \quad \mu_{2}\left(u, \chi_{31}, *\right)=\frac{1}{26}\left(\gamma_{5}+k_{3}\right) \geq 0 ; \\
\mu_{3}\left(u, \chi_{31}, *\right)=\frac{1}{26}\left(-\gamma_{5}+k_{4}\right) \geq 0
\end{gathered}
$$

where $\left(m_{1}, m_{2}, m_{3}, m_{4}, m_{5}\right)=(0,1,1,0,0)$ when $\left(k_{1}, k_{2}, k_{3}, k_{4}\right)=(3993,3950,3980$, 3963), $\left(m_{1}, m_{2}, m_{3}, m_{4}, m_{5}\right)=(0,1,0,1,0)$ when $\left(k_{1}, k_{2}, k_{3}, k_{4}\right)=(3980,3963,3993$, 3950), $\left(m_{1}, m_{2}, m_{3}, m_{4}, m_{5}\right)=(0,1,0,0,1)$ when $\left(k_{1}, k_{2}, k_{3}, k_{4}\right)=(3980,3950,3980$, $3950),\left(m_{1}, m_{2}, m_{3}, m_{4}, m_{5}\right)=(0,1,1,-1,1)$ when $\left(k_{1}, k_{2}, k_{3}, k_{4}\right)=(3993,3937,3967$, 3963), $\left(m_{1}, m_{2}, m_{3}, m_{4}, m_{5}\right)=(0,1,1,1,-1)$ when $\left(k_{1}, k_{2}, k_{3}, k_{4}\right)=(3993,3963,3993$, $3963)$ and $\left(m_{1}, m_{2}, m_{3}, m_{4}, m_{5}\right)=(0,1,-1,1,1)$ when $\left(k_{1}, k_{2}, k_{3}, k_{4}\right)=(3967,3963$, $3993,3937)$. It follows that there are no possible integer solutions for $\left(v_{2 a}, v_{2 b}, v_{13 a}, v_{13 b}\right.$, $\left.v_{13 c}\right)$ in all cases.

Case $(v i)$. Let $u \in V(\mathbb{Z} G)$ where $|u|=39$. Using Propositions 2 and 3,

$$
v_{3 a}+v_{3 b}+v_{13 a}+v_{13 b}+v_{13 c}=1 .
$$

Consider the cases $\chi\left(u^{13}\right)=m_{1} \chi(3 a)+m_{2} \chi(3 b)$ and $\chi\left(u^{3}\right)=m_{3} \chi(13 a)+m_{4} \chi(13 b)+$ $m_{5} \chi(13 c)$ where

$$
\begin{aligned}
\left(m_{1}, m_{2}, m_{3}, m_{4}, m_{5}\right) \in\{ & (1,0,1,0,0),(1,0,0,1,0),(1,0,0,0,1),(0,1,1,0,0), \\
& (0,1,0,1,0),(0,1,0,0,1),(1,0,1,-1,1),(0,1,1,-1,1), \\
& (1,0,1,1,-1),(0,1,1,1,-1),(1,0,-1,1,1),(0,1,-1,1,1), \\
& (3,-2,1,0,0),(3,-2,0,1,),(3,-2,0,0,1),(3,-2,1,-1,1), \\
& (3,-2,1,1,-1),(3,-2,-1,1,1),(2,-1,1,0,0), \\
& (2,-1,0,1,0),(2,-1,0,0,1),(2,-1,1,-1,1), \\
& (2,-1,1,1,-1),(2,-1,-1,1,1),(-1,2,1,0,0), \\
& (-1,2,0,1,0),(-1,2,0,0,1),(-1,2,1,-1,1), \\
& (-1,2,1,1,-1),(-1,2,-1,1,1)\} .
\end{aligned}
$$

Applying Proposition 5, we obtain:

$$
\begin{gathered}
\mu_{13}\left(u, \chi_{2}, *\right)=\frac{1}{39}(12 \gamma+27) \geq 0 ; \quad \mu_{0}\left(u, \chi_{2}, *\right)=\frac{1}{39}(-24 \gamma+24) \geq 0 ; \\
\mu_{1}\left(u, \chi_{2}, *\right)=\frac{1}{39}(-\gamma+27) \geq 0
\end{gathered}
$$

where $\gamma=\nu_{3 a}+\nu_{3 b}$. It follows that there are no possible integer solutions for $\left(v_{3 a}, v_{3 b}, v_{13 a}, v_{13 b}, v_{13 c}\right)$.

Case (vii). Let $u \in V(\mathbb{Z} G)$ where $|u|=91$. Using Propositions 2 and 3,

$$
v_{7 a}+v_{7 b}+v_{7 c}+v_{7 d}+v_{13 a}+v_{13 b}+v_{13 c}=1 \text {. }
$$

Let $\gamma=5 v_{7 a}+5 v_{7 b}+5 v_{7 c}-2 v_{7 d}$. We shall now separately consider the following cases involving $\chi\left(u^{n}\right)$ for $n \in\{7,13\}$ :

- $\chi\left(u^{13}\right)=\chi(7 k)$ and $\chi\left(u^{7}\right)=m_{1} \chi(13 a)+m_{2} \chi(13 b)+m_{3} \chi(13 c)$ where $k \in\{a, b, c\}$ and $\left(m_{1}, m_{2}, m_{3}\right) \in\{(1,0,0),(0,1,0),(0,0,1),(1,-1,1),(1,1,-1),(-1,1,1)\}$. 
Applying Proposition 5, we obtain:

$$
\mu_{0}\left(u, \chi_{2}, *\right)=\frac{1}{91}(72 \gamma+56) \geq 0 ; \quad \mu_{13}\left(u, \chi_{2}, *\right)=\frac{1}{91}(-12 \gamma+21) \geq 0 .
$$

It follows that there are no possible integer values for $\left(v_{7 a}, v_{7 b}, v_{7 c}, v_{7 d}, v_{13 a}, v_{13 b}, v_{13 c}\right)$.

- $\chi\left(u^{13}\right)=\chi(7 d)$ and $\chi\left(u^{7}\right)=m_{1} \chi(13 a)+m_{2} \chi(13 b)+m_{3} \chi(13 c)$ where

$$
\left(m_{1}, m_{2}, m_{3}\right) \in\{(1,0,0),(0,1,0),(0,0,1),(1,-1,1),(1,1,-1),(-1,1,1)\} .
$$

Applying Proposition 5, we obtain:

$$
\mu_{0}\left(u, \chi_{2}, *\right)=\frac{1}{91}(72 \gamma+14) \geq 0 ; \quad \mu_{7}\left(u, \chi_{2}, *\right)=\frac{1}{91}(-6 \gamma+14) \geq 0 .
$$

It follows that there are no possible integer values for $\left(v_{7 a}, v_{7 b}, v_{7 c}, v_{7 d}, v_{13 a}, v_{13 b}, v_{13 c}\right)$.

We shall now consider the prime graph of $G={ }^{3} D_{4}\left(2^{3}\right) . G$ contains elements of order 6 , 14 and 21 . Therefore [2, 3], [2, 7] and [3, 7] are adjacent in $\pi(G)$ and consequently adjacent in $\pi(V(\mathbb{Z} G))$. Clearly $\pi(G)=\pi(V(\mathbb{Z} G))$, since there are no torsion units of order 26,39 and 91 in $V(\mathbb{Z} G)$. This completes the proof.

\section{Proof of Theorem 2}

Let $G={ }^{2} F_{4}(2)^{\prime}$. Clearly $|G|=17971200=2^{11} \cdot 3^{3} \cdot 5^{2} \cdot 13$ and $\exp (G)=3120=$ $2^{4} \cdot 3 \cdot 5 \cdot 13$. Initially, for any torsion unit of $V(\mathbb{Z} G)$ of order $k$ :

$$
\begin{aligned}
v_{2 a} & +v_{2 b}+v_{3 a}+v_{4 a}+v_{4 b}+v_{4 c}+v_{5 a}+v_{6 a}+v_{8 a}+v_{8 b}+v_{8 c}+v_{8 d} \\
& +v_{10 a}+v_{12 a}+v_{12 b}+v_{13 a}+v_{13 b}+v_{16 a}+v_{16 b}+v_{16 c}+v_{16 d}=1
\end{aligned}
$$

For the purpose of this paper, we shall consider torsion units of $V(\mathbb{Z} G)$ of order 2, 3, 5, 10, 15, 26, 39 and 65. We shall now consider each case separately.

Case $(i)$. Let $u \in V(\mathbb{Z} G)$ where $|u|=2$. Using Propositions 2 and 3,

$$
v_{2 a}+v_{2 b}=1 .
$$

Applying Proposition 5, we obtain the following system of inequalities:

$$
\mu_{0}\left(u, \chi_{2}, *\right)=\frac{1}{2}(-2 \gamma+26) \geq 0 ; \quad \mu_{1}\left(u, \chi_{2}, *\right)=\frac{1}{2}(2 \gamma+26) \geq 0
$$

where $\gamma=3 v_{2 a}-v_{2 b}$. It follows that the only possible integer solutions for $\left(v_{2 a}, v_{2 b}\right)$ are listed in part (iii) of Theorem 2.

Case $(i i)$. Let $u \in V(\mathbb{Z} G)$ where $|u|=3$. By Proposition 2, $v_{k x}=0$ for all

$k x \in\{2 a, 2 b, 4 a, 4 b, 4 c, 5 a, 6 a, 8 a, 8 b, 8 c, 8 d, 10 a, 12 a, 12 b, 13 a, 13 b, 16 a, 16 b, 16 c, 16 d\}$.

Therefore, $u$ is rationally conjugated to some element $g \in G$ by Proposition 4 .

Case (iii). Let $u \in V(\mathbb{Z} G)$ where $|u|=5$. By Proposition 2, $v_{k x}=0$ for all

$k x \in\{2 a, 2 b, 3 a, 4 a, 4 b, 4 c, 6 a, 8 a, 8 b, 8 c, 8 d, 10 a, 12 a, 12 b, 13 a, 13 b, 16 a, 16 b, 16 c, 16 d\}$.

Therefore, $u$ is rationally conjugated to some element $g \in G$ by Proposition 4 .

Case $(i v)$. Let $u \in V(\mathbb{Z} G)$ where $|u|=10$. Using Propositions 2 and 3,

$$
v_{2 a}+v_{2 b}+v_{5 a}+v_{10 a}=1 .
$$

Let $\gamma_{1}=6 v_{2 a}-2 v_{2 b}-v_{5 a}+v_{10 a}, \gamma_{2}=14 v_{2 a}-2 v_{2 b}+3 v_{5 a}-v_{10 a}, \gamma_{3}=5 v_{2 a}+v_{2 b}$, $\gamma_{4}=5 v_{2 a}-11 v_{2 b}$ and $\gamma_{5}=32 v_{2 a}+15 v_{2 b}+v_{5 a}+v_{10 a}$. We shall now separately consider the following cases involving $\chi\left(u^{n}\right)$ for $n \in\{2,5\}$ : 
- $\chi\left(u^{5}\right)=m_{1} \chi(2 a)+m_{2} \chi(2 b)$ and $\chi\left(u^{2}\right)=\chi(5 a)$ where $\left(m_{1}, m_{2}\right) \in\{(1,0)$, $(0,1),(-1,2)\}$. Applying Proposition 5, we obtain:

$$
\begin{aligned}
& \mu_{0}\left(u, \chi_{2}, *\right)=\frac{1}{10}\left(-4 \gamma_{1}+k_{1}\right) \geq 0 ; \quad \mu_{1}\left(u, \chi_{2}, *\right)=\frac{1}{10}\left(-\gamma_{1}+k_{2}\right) \geq 0 \\
& \mu_{5}\left(u, \chi_{2}, *\right)=\frac{1}{10}\left(4 \gamma_{1}+k_{3}\right) \geq 0 ; \quad \mu_{0}\left(u, \chi_{6}, *\right)=\frac{1}{10}\left(4 \gamma_{2}+k_{4}\right) \geq 0 ; \\
& \mu_{1}\left(u, \chi_{6}, *\right)=\frac{1}{10}\left(\gamma_{2}+k_{5}\right) \geq 0 ; \quad \mu_{5}\left(u, \chi_{6}, *\right)=\frac{1}{10}\left(-4 \gamma_{2}+k_{6}\right) \geq 0 \\
& \mu_{0}\left(u, \chi_{7}, *\right)=\frac{1}{10}\left(-16 \gamma_{3}+k_{7}\right) \geq 0 ; \quad \mu_{5}\left(u, \chi_{7}, *\right)=\frac{1}{10}\left(16 \gamma_{3}+k_{8}\right) \geq 0 \\
& \mu_{0}\left(u, \chi_{9}, *\right)=\frac{1}{10}\left(4 \gamma_{5}+k_{9}\right) \geq 0 ; \quad \mu_{5}\left(u, \chi_{9}, *\right)=\frac{1}{10}\left(-4 \gamma_{5}+k_{10}\right) \geq 0
\end{aligned}
$$

where the values for $k_{i}$ 's and corresponding $\left(m_{1}, m_{2}\right)$ values are as follows:

\begin{tabular}{ll}
\hline$\left(m_{1}, m_{2}\right)$ & $\left(k_{1}, k_{2}, k_{3}, k_{4}, k_{5}, k_{6}, k_{7}, k_{8}, k_{9}, k_{10}\right)$ \\
\hline$(1,0)$ & $(24,31,36,104,61,76,280,320,386,324)$ \\
$(0,1)$ & $(32,23,28,88,77,92,296,304,370,340)$ \\
$(-1,2)$ & $(40,15,20,72,93,108,312,288,354,356)$ \\
\hline
\end{tabular}

It follows that the only possible integer solutions for $\left(v_{2 a}, v_{2 b}, v_{13 a}\right)$ are $(0,0,5,-4)$, $(0,0,0,1),(0,-4,5,0),(1,1,0,-1),(-1,1,0,1),(-1,-3,0,5),(-1,-3,5,0),(2,2,0$, $-3)$ and $(0,2,0,-1)$ in all cases.

- $\chi\left(u^{5}\right)=3 \chi(2 a)-2 \chi(2 b)$ and $\chi\left(u^{2}\right)=\chi(5 a)$. Applying Proposition 5, we obtain:

$$
\begin{gathered}
\mu_{2}\left(u, \chi_{2}, *\right)=\frac{1}{10}\left(\gamma_{1}+3\right) \geq 0 ; \quad \mu_{0}\left(u, \chi_{2}, *\right)=\frac{1}{10}\left(-4 \gamma_{1}+8\right) \geq 0 ; \\
\mu_{1}\left(u, \chi_{6}, *\right)=\frac{1}{10}\left(\gamma_{2}+29\right) \geq 0 ; \quad \mu_{5}\left(u, \chi_{6}, *\right)=\frac{1}{10}\left(-4 \gamma_{2}+44\right) \geq 0 ; \\
\mu_{5}\left(u, \chi_{7}, *\right)=\frac{1}{10}\left(16 \gamma_{3}+352\right) \geq 0 ; \quad \mu_{0}\left(u, \chi_{7}, *\right)=\frac{1}{10}\left(-16 \gamma_{3}+248\right) \geq 0 ; \\
\mu_{0}\left(u, \chi_{9}, *\right)=\frac{1}{10}\left(4 \gamma_{5}+418\right) \geq 0 .
\end{gathered}
$$

Clearly $\gamma_{1}=-3, \gamma_{2} \in\{-29,-19,-9,1,11\}$ and $\gamma_{3} \in\{3+5 k \mid-5 \leq k \leq 2\}$. It follows that the only possible integer solutions for $\left(v_{2 a}, v_{2 b}, v_{13 a}\right)$ are $(-2,-2,0,5)$ and $(1,3,0,-3)$.

- $\chi\left(u^{5}\right)=2 \chi(2 a)-\chi(2 b)$ and $\chi\left(u^{2}\right)=\chi(5 a)$. Applying Proposition 5, we obtain:

$$
\begin{gathered}
\mu_{2}\left(u, \chi_{2}, *\right)=\frac{1}{10}\left(\gamma_{1}+11\right) \geq 0 ; \quad \mu_{0}\left(u, \chi_{2}, *\right)=\frac{1}{10}\left(-4 \gamma_{1}+16\right) \geq 0 \\
\mu_{0}\left(u, \chi_{6}, *\right)=\frac{1}{10}\left(4 \gamma_{2}+120\right) \geq 0 ; \quad \mu_{5}\left(u, \chi_{6}, *\right)=\frac{1}{10}\left(-4 \gamma_{2}+60\right) \geq 0 ; \\
\mu_{1}\left(u, \chi_{6}, *\right)=\frac{1}{10}\left(\gamma_{2}+45\right) \geq 0 ; \quad \mu_{5}\left(u, \chi_{7}, *\right)=\frac{1}{10}\left(16 \gamma_{3}+336\right) \geq 0 \\
\mu_{0}\left(u, \chi_{7}, *\right)=\frac{1}{10}\left(-16 \gamma_{3}+264\right) \geq 0 ; \quad \mu_{0}\left(u, \chi_{8}, *\right)=\frac{1}{10}\left(4 \gamma_{4}+346\right) \geq 0 ; \\
\mu_{0}\left(u, \chi_{9}, *\right)=\frac{1}{10}\left(4 \gamma_{5}+402\right) \geq 0 .
\end{gathered}
$$

Clearly $\gamma_{1} \in\{-1,-11\}, \gamma_{2} \in\{-25,-15,-5,5,15\}$ and $\gamma_{3} \in\{4+5 k \mid-5 \leq k \leq 2\}$. It follows that the only possible integer solutions for $\left(v_{2 a}, v_{2 b}, v_{13 a}\right)$ are $(-1,-1,0,3)$, $(-1,-1,5,-2),(0,4,-5,2)$ and $(0,4,0,-3)$. 
- $\chi\left(u^{5}\right)=-2 \chi(2 a)+3 \chi(2 b)$ and $\chi\left(u^{2}\right)=\chi(5 a)$. Applying Proposition 5, we obtain:

$$
\begin{gathered}
\mu_{5}\left(u, \chi_{2}, *\right)=\frac{1}{10}\left(4 \gamma_{1}+12\right) \geq 0 ; \quad \mu_{1}\left(u, \chi_{2}, *\right)=\frac{1}{10}\left(-\gamma_{1}+7\right) \geq 0 ; \\
\mu_{0}\left(u, \chi_{6}, *\right)=\frac{1}{10}\left(4 \gamma_{2}+56\right) \geq 0 ; \quad \mu_{5}\left(u, \chi_{6}, *\right)=\frac{1}{10}\left(-4 \gamma_{2}+124\right) \geq 0 ; \\
\mu_{1}\left(u, \chi_{6}, *\right)=\frac{1}{10}\left(\gamma_{2}+109\right) \geq 0 ; \quad \mu_{5}\left(u, \chi_{7}, *\right)=\frac{1}{10}\left(16 \gamma_{3}+272\right) \geq 0 ; \\
\mu_{0}\left(u, \chi_{7}, *\right)=\frac{1}{10}\left(-16 \gamma_{3}+328\right) \geq 0 ; \quad \mu_{0}\left(u, \chi_{8}, *\right)=\frac{1}{10}\left(\gamma_{4}+282\right) \geq 0 ; \\
\mu_{0}\left(u, \chi_{9}, *\right)=\frac{1}{10}\left(\gamma_{5}+338\right) \geq 0 .
\end{gathered}
$$

Clearly $\gamma_{1} \in\{-3,7\} \gamma_{2} \in\{-9,1,11,21,3\}$ and $\gamma_{3} \in\{3+5 k \mid-4 \leq k \leq\}$. It follows that the only possible integer solutions for $\left(v_{2 a}, v_{2 b}, v_{13 a}\right)$ are $(1,3,0,-3),(0,-2,5,-2)$, $(1,3,-5,2)$ and $(0,-2,0,3)$.

- $\chi\left(u^{5}\right)=-3 \chi(2 a)+4 \chi(2 b)$ and $\chi\left(u^{2}\right)$. Applying Proposition 5, we obtain:

$$
\begin{aligned}
& \mu_{5}\left(u, \chi_{2}, *\right)=\frac{1}{10}\left(4 \gamma_{1}+4\right) \geq 0 ; \quad \mu_{1}\left(u, \chi_{2}, *\right)=\frac{1}{10}\left(-\gamma_{1}-1\right) \geq 0 ; \\
& \mu_{0}\left(u, \chi_{6}, *\right)=\frac{1}{10}\left(4 \gamma_{2}+40\right) \geq 0 ; \quad \mu_{2}\left(u, \chi_{6}, *\right)=\frac{1}{10}\left(-\gamma_{2}+25\right) \geq 0 ; \\
& \mu_{5}\left(u, \chi_{7}, *\right)=\frac{1}{10}\left(16 \gamma_{3}+256\right) \geq 0 ; \quad \mu_{0}\left(u, \chi_{7}, *\right)=\frac{1}{10}\left(-16 \gamma_{3}+344\right) \geq 0 ; \\
& \mu_{0}\left(u, \chi_{9}, *\right)=\frac{1}{10}\left(4 \gamma_{5}+322\right) \geq 0 ; \quad \mu_{5}\left(u, \chi_{9}, *\right)=\frac{1}{10}\left(-4 \gamma_{5}+388\right) \geq 0 .
\end{aligned}
$$

Clearly $\gamma_{1}=-1, \gamma_{2} \in\{-5,5,15,25\}$ and $\gamma_{3} \in\{4+5 k \mid-4 \leq k \leq 3\}$. It follows that there are no possible integer solutions for $\left(v_{2 a}, v_{2 b}, v_{13 a}\right)$ in this case.

Case $(v)$. Let $u \in V(\mathbb{Z} G)$ where $|u|=13$. Using Propositions 2 and 3 ,

$$
v_{13 a}+v_{13 b}=1 .
$$

Applying Proposition 5, we obtain the following system of inequalities:

$$
\begin{gathered}
\mu_{1}\left(u, \chi_{7}, 5\right)=\frac{1}{13}\left(\gamma_{1}+109\right) \geq 0 ; \quad \mu_{2}\left(u, \chi_{15}, 5\right)=\frac{1}{13}\left(-\gamma_{1}+593\right) \geq 0 ; \\
\mu_{2}\left(u, \chi_{7}, 5\right)=\frac{1}{13}\left(\gamma_{2}+109\right) \geq 0
\end{gathered}
$$

where $\gamma_{1}=8 v_{13 a}-5 v_{13 b}$ and $\gamma_{2}=-5 v_{13 a}+8 v_{13 b}$. It follows that the only possible integer solutions for $\left(v_{3 a}, v_{3 b}\right)$ are listed in part $(v)$ of Theorem 2.

Case (vi). Let $u \in V(\mathbb{Z} G)$ where $|u|=15$. Using Propositions 2 and 3,

$$
v_{3 a}+v_{5 a}=1 .
$$

Applying Proposition 5, we obtain the following system of inequalities:

$$
\mu_{0}\left(u, \chi_{2}, *\right)=\frac{1}{15}(-8 \gamma+28) \geq 0 ; \quad \mu_{5}\left(u, \chi_{2}, *\right)=\frac{1}{15}(4 \gamma+31) \geq 0
$$

where $\gamma=\nu_{3 a}-v_{5 a}$. Clearly $\gamma=-4$ and there are no possible integer solutions for $\left(v_{3 a}, v_{5 a}\right)$.

Case (vii). Let $u \in V(\mathbb{Z} G)$ where $|u|=26$. Using Propositions 2 and 3,

$$
v_{2 a}+v_{2 b}+v_{13 a}+v_{13 b}=1 \text {. }
$$

Let $\gamma_{1}=3 v_{2 a}-v_{2 b}, \gamma_{2}=7 v_{2 a}-v_{2 b}, \gamma_{3}=-20 v_{2 a}-4 v_{2 b}+v_{13 a}+v_{13 b}$ and $\gamma_{4}=$ $6 v_{13 a}-7 v_{13 b}$. We shall now separately consider the following cases involving $\chi\left(u^{n}\right)$ for $n \in\{2,13\}:$

- $\chi\left(u^{13}\right)=m_{1} \chi(2 a)+m_{2} \chi(2 b)$ and $\chi\left(u^{2}\right)=m_{3} \chi(5 a)+m_{4} \chi(5 b)$ where $\left(m_{1}, m_{2}, m_{3}\right.$, $\left.m_{4}\right) \in\{(1,0,1,0),(1,0,0,1),(1,0,9,-8),(1,0,8,-7),(1,0,7,-6),(1,0,6,-5)$, $(1,0,5,-4),(1,0,4,-3),(1,0,3,-2),(1,0,2,-1),(1,0,-1,2),(1,0,-2,3),(1$, 
$0,-3,4),(1,0,-4,5),(1,0,-5,6),(1,0,-6,7),(1,0,-7,8),(1,0,-8,9)\}$. Applying Proposition 5, we obtain:

$$
\mu_{13}\left(u, \chi_{2}, *\right)=\frac{1}{26}\left(24 \gamma_{1}+32\right) \geq 0 ; \quad \mu_{0}\left(u, \chi_{2}, *\right)=\frac{1}{26}\left(-24 \gamma_{1}+20\right) \geq 0 .
$$

It follows that there are no possible integer values for $\left(v_{2 a}, v_{2 b}, v_{13 a}, v_{13 b}\right)$.

- $\chi\left(u^{13}\right)=m_{1} \chi(2 a)+m_{2} \chi(2 b)$ and $\chi\left(u^{2}\right)=m_{3} \chi(5 a)+m_{4} \chi(5 b)$ where $\left(m_{1}, m_{2}, m_{3}\right.$, $\left.m_{4}\right) \in\{(2,-1,1,0),(2,-1,0,1),(2,-1,9,-8),(2,-1,8,-7),(2,-1,7,-6),(2$, $-1,6,-5),(2,-1,5,-4),(2,-1,4,-3),(2,-1,3,-2),(2,-1,2-1),(2,-1,-1$, $2),(2,-1,-2,3),(2,-1,-3,4),(2,-1,-4,5),(2,-1,-5,6),(2,-1,-6,7),(2$, $-1,-7,8),(2,-1,-8,9)\}$. Applying Proposition 5, we obtain:

$$
\mu_{13}\left(u, \chi_{2}, *\right)=\frac{1}{26}\left(24 \gamma_{1}+16\right) \geq 0 ; \quad \mu_{0}\left(u, \chi_{2}, *\right)=\frac{1}{26}\left(-24 \gamma_{1}+36\right) \geq 0 .
$$

It follows that there are no possible integer values for $\left(v_{2 a}, v_{2 b}, v_{13 a}, v_{13 b}\right)$.

- $\chi\left(u^{13}\right)=m_{1} \chi(2 a)+m_{2} \chi(2 b)$ and $\chi\left(u^{2}\right)=m_{3} \chi(5 a)+m_{4} \chi(5 b)$ where $\left(m_{1}, m_{2}, m_{3}\right.$, $\left.m_{4}\right) \in\{(-2,3,1,0),(-2,3,0,1),(-2,3,9,-8),(-2,3,8,-7),(-2,3,7,-6),(-2$, $3,6,-5),(-2,3,5,-4),(-2,3,4,-3),(-2,3,3,-2),(-2,3,2,-1),(-2,3,-1,2)$, $(-2,3,-2,3),(-2,3,-3,4),(-2,3,-4,5),(-2,3,-5,6),(-2,3,-6,7),(-2,3$, $-7,8),(-2,3,-8,9)\}$. Applying Proposition 5, we obtain:

$$
\mu_{13}\left(u, \chi_{2}, *\right)=\frac{1}{26}\left(24 \gamma_{1}+8\right) \geq 0 ; \quad \mu_{0}\left(u, \chi_{2}, *\right)=\frac{1}{26}\left(-24 \gamma_{1}+44\right) \geq 0 .
$$

It follows that there are no possible integer values for $\left(v_{2 a}, v_{2 b}, v_{13 a}, v_{13 b}\right)$.

- $\chi\left(u^{13}\right)=m_{1} \chi(2 a)+m_{2} \chi(2 b)$ and $\chi\left(u^{2}\right)=m_{3} \chi(5 a)+m_{4} \chi(5 b)$ where $\left(m_{1}, m_{2}, m_{3}\right.$, $\left.m_{4}\right) \in\{(0,1,9,-8),(0,1,-8,9)\}$. Applying Proposition 5, we obtain:

$$
\begin{gathered}
\mu_{13}\left(u, \chi_{2}, *\right)=\frac{1}{26}\left(24 \gamma_{1}+24\right) \geq 0 ; \quad \mu_{0}\left(u, \chi_{2}, *\right)=\frac{1}{26}\left(-24 \gamma_{1}+28\right) \geq 0 ; \\
\mu_{0}\left(u, \chi_{6}, *\right)=\frac{1}{26}\left(24 \gamma_{2}+76\right) \geq 0 ; \quad \mu_{13}\left(u, \chi_{6}, *\right)=\frac{1}{26}\left(-24 \gamma_{2}+80\right) \geq 0 ; \\
\mu_{1}\left(u, \chi_{7}, *\right)=\frac{1}{26}\left(\gamma_{3}+303\right) \geq 0 .
\end{gathered}
$$

Clearly $\gamma_{1}=\gamma_{2}=-1$. It follows that there are no possible integer values for $\left(\nu_{2 a}\right.$, $\left.v_{2 b}, v_{13 a}, v_{13 b}\right)$.

- $\chi\left(u^{13}\right)=m_{1} \chi(2 a)+m_{2} \chi(2 b)$ and $\chi\left(u^{2}\right)=m_{3} \chi(5 a)+m_{4} \chi(5 b)$ where $\left(m_{1}, m_{2}, m_{3}\right.$, $\left.m_{4}\right) \in\{(3,-2,9,-8),(3,-2,-8,9)\}$. Applying Proposition 5, we obtain:

$$
\begin{aligned}
& \mu_{2}\left(u, \chi_{2}, *\right)=\frac{1}{26}\left(2 \gamma_{1}+4\right) \geq 0 ; \quad \mu_{0}\left(u, \chi_{2}, *\right)=\frac{1}{26}\left(-24 \gamma_{1}+4\right) \geq 0 ; \\
& \mu_{0}\left(u, \chi_{6}, *\right)=\frac{1}{26}\left(24 \gamma_{2}+124\right) \geq 0 ; \quad \mu_{13}\left(u, \chi_{6}, *\right)=\frac{1}{26}\left(-24 \gamma_{2}+32\right) \geq 0 .
\end{aligned}
$$

Clearly $\gamma_{1}=-2$ and $\gamma_{2}=-3$. It follows that there are no possible integer values for $\left(v_{2 a}, v_{2 b}, v_{13 a}, v_{13 b}\right)$.

- $\chi\left(u^{13}\right)=m_{1} \chi(2 a)+m_{2} \chi(2 b)$ and $\chi\left(u^{2}\right)=m_{3} \chi(5 a)+m_{4} \chi(5 b)$ where $\left(m_{1}, m_{2}, m_{3}\right.$, $\left.m_{4}\right) \in\{(-3,4,9,-8),(-3,4,-8,9)\}$. Applying Proposition 5, we obtain:

$$
\begin{aligned}
\mu_{13}\left(u, \chi_{2}, *\right) & =\frac{1}{26}\left(24 \gamma_{1}\right) \geq 0 ; \quad \mu_{1}\left(u, \chi_{2}, *\right)=\frac{1}{26}\left(-2 \gamma_{1}\right) \geq 0 ; \\
\mu_{0}\left(u, \chi_{6}, *\right) & =\frac{1}{26}\left(24 \gamma_{2}+28\right) \geq 0 ; \quad \mu_{13}\left(u, \chi_{6}, *\right)=\frac{1}{26}\left(-24 \gamma_{2}+128\right) \geq 0 .
\end{aligned}
$$

Clearly $\gamma_{1}=0$ and $\gamma_{2}=1$. It follows that there are no possible integer values for $\left(v_{2 a}, v_{2 b}, v_{13 a}, v_{13 b}\right)$. 
- $\chi\left(u^{13}\right)=m_{1} \chi(2 a)+m_{2} \chi(2 b)$ and $\chi\left(u^{2}\right)=m_{3} \chi(5 a)+m_{4} \chi(5 b)$ where $\left(m_{1}, m_{2}, m_{3}\right.$, $\left.m_{4}\right) \in\{(0,1,1,0),(0,1,0,1),(0,1,8,-7),(0,1,7,-6),(0,1,6,-5),(0,1,5,-4)$, $(0,1,4,-3), \quad(0,1,3,-2),(0,1,2,-1),(0,1,-1,2),(0,1,-2,3),(0,1,-3,4)$, $(0,1,-4,5),(0,1,-5,6),(0,1,-6,7),(0,1,-7,8)\}$. Applying Proposition 5, we obtain:

$$
\begin{gathered}
\mu_{13}\left(u, \chi_{2}, *\right)=\frac{1}{26}\left(24 \gamma_{1}+24\right) \geq 0 ; \quad \mu_{0}\left(u, \chi_{2}, *\right)=\frac{1}{26}\left(-24 \gamma_{1}+28\right) \geq 0 ; \\
\mu_{0}\left(u, \chi_{6}, *\right)=\frac{1}{26}\left(24 \gamma_{2}+76\right) \geq 0 ; \quad \mu_{13}\left(u, \chi_{6}, *\right)=\frac{1}{26}\left(-24 \gamma_{2}+80\right) \geq 0 ; \\
\mu_{1}\left(u, \chi_{7}, *\right)=\frac{1}{26}\left(\gamma_{3}+303\right) \geq 0 ; \quad \mu_{4}\left(u, \chi_{21}, *\right)=\frac{1}{26}\left(\gamma_{4}+k\right) \geq 0 ; \\
\mu_{1}\left(u, \chi_{21}, *\right)=\frac{1}{26}\left(-\gamma_{4}+k\right) \geq 0
\end{gathered}
$$

where the corresponding $k$ values to the above cases are:

\begin{tabular}{lllr}
\hline$\left(m_{1}, m_{2}, m_{3}, m_{4}\right)$ & $k$ & $\left(m_{1}, m_{2}, m_{3}, m_{4}\right)$ & $k$ \\
\hline$(0,1,1,0)$ & 2041 & $(0,1,0,1)$ & 2054 \\
$(0,1,8,-7)$ & 1950 & $(0,1,7,-6)$ & 1963 \\
$(0,1,6,-5)$ & 1976 & $(0,1,5,-4)$ & 1989 \\
$(0,1,4,-3)$ & 2002 & $(0,1,3,-2)$ & 2015 \\
$(0,1,2,-1)$ & 2028 & $(0,1,-1,2)$ & 2067 \\
$(0,1,-2,3)$ & 2080 & $(0,1,-3,4)$ & 2093 \\
$(0,1,-4,5)$ & 2106 & $(0,1,-5,6)$ & 2119 \\
$(0,1,-6,7)$ & 2132 & $(0,1,-7,8)$ & 2145 \\
\hline
\end{tabular}

It follows that there are no possible integer values for $\left(v_{2 a}, v_{2 b}, v_{13 a}, v_{13 b}\right)$ in all cases.

- $\chi\left(u^{13}\right)=m_{1} \chi(2 a)+m_{2} \chi(2 b)$ and $\chi\left(u^{2}\right)=m_{3} \chi(5 a)+m_{4} \chi(5 b)$ where $\left(m_{1}, m_{2}, m_{3}\right.$, $\left.m_{4}\right) \in\{(3,-2,1,0),(3,-2,0,1),(3,-2,8,-7),(3,-2,7,-6),(3,-2,6,-5)$, $(3,-2,5,-4),(3,-2,4,-3),(3,-2,3,-2),(3,-2,2,-1),(3,-2,-1,2),(3,-2$, $-2,3),(3,-2,-3,4),(3,-2,-4,5),(3,-2,-5,6),(3,-2,-6,7),(3,-2,-7,8)\}$. Applying Proposition 5, we obtain:

$$
\begin{aligned}
\mu_{2}\left(u, \chi_{2}, *\right) & =\frac{1}{26}\left(2 \gamma_{1}+4\right) \geq 0 ; \quad \mu_{0}\left(u, \chi_{2}, *\right)=\frac{1}{26}\left(-24 \gamma_{1}+4\right) \geq 0 ; \\
\mu_{0}\left(u, \chi_{6}, *\right) & =\frac{1}{26}\left(24 \gamma_{2}+124\right) \geq 0 ; \quad \mu_{13}\left(u, \chi_{6}, *\right)=\frac{1}{26}\left(-24 \gamma_{2}+32\right) \geq 0 ; \\
\mu_{4}\left(u, \chi_{21}, *\right) & =\frac{1}{26}\left(\gamma_{4}+k\right) \geq 0 ; \quad \mu_{1}\left(u, \chi_{21}, *\right)=\frac{1}{26}\left(-\gamma_{4}+k\right) \geq 0 .
\end{aligned}
$$

where the corresponding $k$-values to the above cases are:

\begin{tabular}{lllr}
\hline$\left(m_{1}, m_{2}, m_{3}, m_{4}\right)$ & $k$ & $\left(m_{1}, m_{2}, m_{3}, m_{4}\right)$ & $k$ \\
\hline$(3,-2,1,0)$ & 2041 & $(3,-2,0,1)$ & 2054 \\
$(3,-2,8,-7)$ & 1950 & $(3,-2,7,-6)$ & 1963 \\
$(3,-2,6,-5)$ & 1976 & $(3,-2,5,-4)$ & 1989 \\
$(3,-2,4,-3)$ & 2002 & $(3,-2,3,-2)$ & 2015 \\
$(3,-2,2,-1)$ & 2028 & $(3,-2,-1,2)$ & 2067 \\
$(3,-2,-2,3)$ & 2080 & $(3,-2,-3,4)$ & 2093 \\
$(3,-2,-4,5)$ & 2106 & $(3,-2,-5,6)$ & 2119 \\
$(3,-2,-6,7)$ & 2132 & $(3,-2,-7,8)$ & 2145 \\
\hline
\end{tabular}


It follows that there are no possible integer values for $\left(v_{2 a}, v_{2 b}, v_{13 a}, v_{13 b}\right)$ in all cases.

$\chi\left(u^{13}\right)=m_{1} \chi(2 a)+m_{2} \chi(2 b)$ and $\chi\left(u^{2}\right)=m_{3} \chi(5 a)+m_{4} \chi(5 b)$ where $\left(m_{1}, m_{2}, m_{3}\right.$, $\left.m_{4}\right) \in\{(-3,4,1,0),(-3,4,0,1),(-3,4,8,-7),(-3,4,7,-6),(-3,4,6,-5),(-3$, $4,5,-4),(-3,4,4,-3),(-3,4,3,-2),(-3,4,2,-1),(-3,4,-1,2),(-3,4,-2,3)$, $(-3,4,-3,4),(-3,4,-4,5),(-3,4,-5,6),(-3,4,-6,7),(-3,4,-7,8)\}$. Applying Proposition 5, we obtain:

$$
\begin{aligned}
\mu_{13}\left(u, \chi_{2}, *\right) & =\frac{1}{26}\left(24 \gamma_{1}\right) \geq 0 ; \quad \mu_{1}\left(u, \chi_{2}, *\right)=\frac{1}{26}\left(-2 \gamma_{1}\right) \geq 0 ; \\
\mu_{0}\left(u, \chi_{6}, *\right) & =\frac{1}{26}\left(24 \gamma_{2}+28\right) \geq 0 ; \quad \mu_{13}\left(u, \chi_{6}, *\right)=\frac{1}{26}\left(-24 \gamma_{2}+128\right) \geq 0 ; \\
\mu_{4}\left(u, \chi_{21}, *\right) & =\frac{1}{26}\left(\gamma_{4}+k\right) \geq 0 ; \quad \mu_{1}\left(u, \chi_{21}, *\right)=\frac{1}{26}\left(-\gamma_{4}+k\right) \geq 0 .
\end{aligned}
$$

where the corresponding $k$ values to the above cases are:

\begin{tabular}{lllr}
\hline$\left(m_{1}, m_{2}, m_{3}, m_{4}\right)$ & $k$ & $\left(m_{1}, m_{2}, m_{3}, m_{4}\right)$ & $k$ \\
\hline$(-3,4,1,0)$ & 2041 & $(-3,4,0,1)$ & 2054 \\
$(-3,4,8,-7)$ & 1950 & $(-3,4,7,-6)$ & 1963 \\
$(-3,4,6,-5)$ & 1976 & $(-3,4,5,-4)$ & 1989 \\
$(-3,4,4,-3)$ & 2002 & $(-3,4,3,-2)$ & 2015 \\
$(-3,4,2,-1)$ & 2028 & $(-3,4,-1,2)$ & 2067 \\
$(-3,4,-2,3)$ & 2080 & $(-3,4,-3,4)$ & 2093 \\
$(-3,4,-4,5)$ & 2106 & $(-3,4,-5,6)$ & 2119 \\
$(-3,4,-6,7)$ & 2132 & $(-3,4,-7,8)$ & 2145 \\
\hline
\end{tabular}

It follows that there are no possible integer values for $\left(v_{2 a}, v_{2 b}, v_{13 a}, v_{13 b}\right)$ in all cases.

Case (viii). Let $u \in V(\mathbb{Z} G)$ where $|u|=39$. Using Propositions 2 and 3,

$$
v_{3 a}+v_{13 a}+v_{13 b}=1 \text {. }
$$

Consider the cases $\chi\left(u^{13}\right)=\chi(3 a)$ and $\chi\left(u^{3}\right)=m_{1} \chi(13 a)+m_{2} \chi(13 b)$ where

$$
\begin{aligned}
\left(m_{1}, m_{2}\right) \in\{ & (1,0),(0,1),(9,-8),(8,-7),(7,-6),(6,-5), \\
& (5,-4),(4,-3),(3,-2),(2,-1),(-1,2),(-2,3), \\
& (-3,4),(-4,5),(-5,6),(-6,7),(-7,8),(-8,9)\} .
\end{aligned}
$$

Applying Proposition 5, we obtain:

$$
\begin{gathered}
\mu_{13}\left(u, \chi_{2}, *\right)=\frac{1}{39}\left(12 v_{3 a}+27\right) \geq 0 ; \quad \mu_{0}\left(u, \chi_{2}, *\right)=\frac{1}{39}\left(-24 v_{3 a}+24\right) \geq 0 ; \\
\mu_{1}\left(u, \chi_{2}, *\right)=\frac{1}{39}\left(-v_{3 a}+27\right) \geq 0 .
\end{gathered}
$$

It follows that there are no possible integer solutions for $\left(v_{3 a}, v_{13 a}, v_{13 b}\right)$.

Case $(i x)$. Let $u \in V(\mathbb{Z} G)$ where $|u|=65$. Using Propositions 2 and 3 ,

$$
v_{5 a}+v_{13 a}+v_{13 b}=1 \text {. }
$$

Consider the cases $\chi\left(u^{13}\right)=\chi(5 a)$ and $\chi\left(u^{5}\right)=m_{1} \chi(13 a)+m_{2} \chi(13 b)$ where

$$
\begin{aligned}
\left(m_{1}, m_{2}\right) \in\{ & (1,0),(0,1),(9,-8),(8,-7),(7,-6),(6,-5), \\
& (5,-4),(4,-3),(3,-2),(2,-1),(-1,2),(-2,3), \\
& (-3,4),(-4,5),(-5,6),(-6,7),(-7,8),(-8,9)\} .
\end{aligned}
$$


Applying Proposition 5, we obtain:

$$
\mu_{0}\left(u, \chi_{2}, *\right)=\frac{1}{65}\left(48 v_{5 a}+30\right) \geq 0 ; \quad \mu_{13}\left(u, \chi_{2}, *\right)=\frac{1}{65}\left(-12 v_{5 a}+25\right) \geq 0 .
$$

It follows that there are no possible integer solutions for $\left(v_{5 a}, v_{13 a}, v_{13 b}\right)$.

We shall now consider the prime graph of $G={ }^{2} F_{4}(2)^{\prime}$. $G$ contains elements of order 6 and 10 . Therefore $[2,3]$ and $[2,5]$ are adjacent in $\pi(G)$ and consequently adjacent in $\pi(V(\mathbb{Z} G))$. Clearly $\pi(G)=\pi(V(\mathbb{Z} G))$, since there are no torsion units of order 15, 26, 39 and 65 in $V(\mathbb{Z} G)$. This completes the proof.

\section{Proof of Theorem 3}

Let $G={ }^{2} F_{4}(2)$. Clearly $|G|=35942400=2^{12} \cdot 3^{3} \cdot 5^{2} \cdot 13$ and $\exp (G)=3120=2^{4} \cdot 3 \cdot 5 \cdot 13$. Initially, for any torsion unit of $V(\mathbb{Z} G)$ of order $k$ :

$$
\begin{aligned}
v_{2 a} & +v_{2 b}+v_{3 a}+v_{4 a}+v_{4 b}+v_{4 c}+v_{5 a}+v_{6 a}+v_{8 a}+v_{8 b}+v_{8 c} \\
& +v_{10 a}+v_{12 a}+v_{13 a}+v_{16 a}+v_{16 b}+v_{4 d}+v_{4 e}+v_{4 f}+v_{4 g} \\
& +v_{8 d}+v_{8 e}+v_{12 b}+v_{12 c}+v_{16 c}+v_{16 d}+v_{20 a}+v_{20 b}=1
\end{aligned}
$$

For the purpose of this paper, we shall consider torsion units of $V(\mathbb{Z} G)$ of order $2,3,5,10$, $15,26,39$ and 65 . We shall now consider each case separately.

Case $(i)$. Let $u \in V(\mathbb{Z} G)$ where $|u|=2$. Using Propositions 2 and 3 ,

$$
v_{2 a}+v_{2 b}=1 \text {. }
$$

Applying Proposition 5, we obtain the following system of inequalities:

$$
\mu_{1}\left(u, \chi_{3}, *\right)=\frac{1}{2}(4 \gamma+52) \geq 0 ; \quad \mu_{0}\left(u, \chi_{3}, *\right)=\frac{1}{2}(-4 \gamma+52) \geq 0
$$

where $\gamma=3 v_{2 a}-v_{2 b}$. Clearly $\gamma \in\{k \mid-13 \leq k \leq 13\}$. It follows that the only possible integer solutions for $\left(v_{2 a}, v_{2 b}\right)$ are listed in part (iii) of Theorem 3.

Case $(i i)$. Let $u \in V(\mathbb{Z} G)$ where $|u|=3$. By Proposition 2, $v_{k x}=0$ for all

$$
\begin{array}{r}
k x \in\{2 a, 2 b, 4 a, 4 b, 4 c, 5 a, 6 a, 8 a, 8 b, 8 c, 10 a, 12 a, 13 a, 16 a, \\
16 b, 4 d, 4 e, 4 f, 4 g, 8 d, 8 e, 12 b, 12 c, 16 c, 16 d, 20 a, 20 b\} .
\end{array}
$$

Therefore, $u$ is rationally conjugated to some element $g \in G$ by Proposition 4 .

Case (iii). Let $u \in V(\mathbb{Z} G)$ where $|u|=5$. By Proposition $2, v_{k x}=0$ for all

$$
\begin{array}{r}
k x \in\{2 a, 2 b, 3 a, 4 a, 4 b, 4 c, 6 a, 8 a, 8 b, 8 c, 10 a, 12 a, 13 a, 16 a, \\
16 b, 4 d, 4 e, 4 f, 4 g, 8 d, 8 e, 12 b, 12 c, 16 c, 16 d, 20 a, 20 b\} .
\end{array}
$$

Therefore, $u$ is rationally conjugated to some element $g \in G$ by Proposition 4 .

Case (iv). Let $u \in V(\mathbb{Z} G)$ where $|u|=10$. Using Propositions 2 and 3,

$$
v_{2 a}+v_{2 b}+v_{5 a}+v_{10 a}=1 .
$$

Let $\gamma_{1}=6 v_{2 a}-2 v_{2 b}-v_{5 a}+v_{10 a}, \gamma_{2}=14 v_{2 a}-2 v_{2 b}+3 v_{5 a}-v_{10 a}, \gamma_{3}=5 v_{2 a}+v_{2 b}$, $\gamma_{4}=5 v_{2 a}-11 v_{2 b}$ and $\gamma_{5}=31 v_{2 a}+15 v_{2 b}+v_{5 a}+v_{10 a}$. We shall now separately consider the following cases involving $\chi\left(u^{n}\right)$ for $n \in\{2,5\}$ : 
- $\chi\left(u^{5}\right)=m_{1} \chi(2 a)+m_{2} \chi(2 b)$ and $\chi\left(u^{2}\right)=\chi(5 a)$ where $\left(m_{1}, m_{2}\right) \in\{(1,0)$, $(0,1),(-1,2)\}$. Applying Proposition 5, we obtain:

$$
\begin{gathered}
\mu_{0}\left(u, \chi_{3}, *\right)=\frac{1}{10}\left(-8 \gamma_{1}+k_{1}\right) \geq 0 ; \quad \mu_{5}\left(u, \chi_{3}, *\right)=\frac{1}{10}\left(8 \gamma_{1}+k_{2}\right) \geq 0 ; \\
\mu_{0}\left(u, \chi_{8}, *\right)=\frac{1}{10}\left(4 \gamma_{2}+k_{3}\right) \geq 0 ; \quad \mu_{1}\left(u, \chi_{8}, *\right)=\frac{1}{10}\left(\gamma_{2}+k_{4}\right) \geq 0 ; \\
\mu_{5}\left(u, \chi_{8}, *\right)=\frac{1}{10}\left(-4 \gamma_{2}+k_{5}\right) \geq 0 ; \quad \mu_{0}\left(u, \chi_{10}, *\right)=\frac{1}{10}\left(-16 \gamma_{3}+k_{6}\right) \geq 0 ; \\
\mu_{5}\left(u, \chi_{10}, *\right)=\frac{1}{10}\left(16 \gamma_{3}+k_{7}\right) \geq 0 ; \quad \mu_{0}\left(u, \chi_{14}, *\right)=\frac{1}{10}\left(4 \gamma_{4}+k_{8}\right) \geq 0 ; \\
\mu_{5}\left(u, \chi_{14}, *\right)=\frac{1}{10}\left(-4 \gamma_{4}+k_{9}\right) \geq 0
\end{gathered}
$$

where the values for $k_{i}$ 's and corresponding $\left(m_{1}, m_{2}\right)$ values are as follows:

\begin{tabular}{ll}
\hline$\left(m_{1}, m_{2}\right)$ & $\left(k_{1}, k_{2}, k_{3}, k_{4}, k_{5}, k_{6}, k_{7}, k_{8}, k_{9}\right)$ \\
\hline$(1,0)$ & $(48,72,104,61,76,280,320,386,324)$ \\
$(0,1)$ & $(64,56,88,77,92,296,304,370,340)$ \\
$(-1,2)$ & $(80,40,72,93,108,312,288,354,356)$
\end{tabular}

It follows that the only possible integer solutions for $\left(v_{2 a}, v_{2 b}, v_{13 a}\right)$ are $(0,0,5,-4)$, $(0,0,0,1),(-1,1,0,1),(1,1,0,-1),(0,-4,5,0),(0,2,0,-1),(-1,-3,5,0),(-1,-3$, $0,5)$ and $(2,2,0,-3)$ in all cases.

- $\chi\left(u^{5}\right)=2 \chi(2 a)-\chi(2 b)$ and $\chi\left(u^{2}\right)=\chi(5 a)$. Applying Proposition 5, we obtain:

$$
\begin{gathered}
\mu_{2}\left(u, \chi_{3}, *\right)=\frac{1}{10}\left(2 \gamma_{1}+22\right) \geq 0 ; \quad \mu_{0}\left(u, \chi_{3}, *\right)=\frac{1}{10}\left(-8 \gamma_{1}+32\right) \geq 0 ; \\
\mu_{0}\left(u, \chi_{8}, *\right)=\frac{1}{10}\left(4 \gamma_{2}+120\right) \geq 0 ; \quad \mu_{5}\left(u, \chi_{8}, *\right)=\frac{1}{10}\left(-4 \gamma_{2}+60\right) \geq 0 ; \\
\mu_{1}\left(u, \chi_{8}, *\right)=\frac{1}{10}\left(\gamma_{2}+45\right) \geq 0 ; \quad \mu_{5}\left(u, \chi_{10}, *\right)=\frac{1}{10}\left(16 \gamma_{3}+336\right) \geq 0 ; \\
\mu_{0}\left(u, \chi_{10}, *\right)=\frac{1}{10}\left(-16 \gamma_{3}+264\right) \geq 0 ; \quad \mu_{0}\left(u, \chi_{12}, *\right)=\frac{1}{10}\left(4 \gamma_{4}+346\right) \geq 0 ; \\
\mu_{0}\left(u, \chi_{14}, *\right)=\frac{1}{10}\left(4 \gamma_{5}+402\right) \geq 0 .
\end{gathered}
$$

Clearly $\gamma_{1} \in\{-11,-6,-1,4\}, \gamma_{2} \in\{4+5 k \mid-5 \leq k \leq 2\}$ and $\gamma_{3} \in\{4+5 k \mid-5 \leq k \leq 2\}$. It follows that the only possible integer solutions for $\left(v_{2 a}, v_{2 b}, v_{13 a}\right)$ are $(-1,-1,0,3)$, $(-1,-1,5,-2),(0,4,-5,2)$ and $(0,4,0,-3)$.

- $\chi\left(u^{5}\right)=-2 \chi(2 a)+3 \chi(2 b)$ and $\chi\left(u^{2}\right)=\chi(5 a)$. Applying Proposition 5, we obtain:

$$
\begin{gathered}
\mu_{5}\left(u, \chi_{3}, *\right)=\frac{1}{10}\left(8 \gamma_{1}+24\right) \geq 0 ; \quad \mu_{1}\left(u, \chi_{3}, *\right)=\frac{1}{10}\left(-2 \gamma_{1}+14\right) \geq 0 ; \\
\mu_{0}\left(u, \chi_{8}, *\right)=\frac{1}{10}\left(4 \gamma_{2}+56\right) \geq 0 ; \quad \mu_{5}\left(u, \chi_{8}, *\right)=\frac{1}{10}\left(-4 \gamma_{2}+124\right) \geq 0 ; \\
\mu_{1}\left(u, \chi_{8}, *\right)=\frac{1}{10}\left(\gamma_{2}+109\right) \geq 0 ; \quad \mu_{5}\left(u, \chi_{10}, *\right)=\frac{1}{10}\left(16 \gamma_{3}+272\right) \geq 0 ; \\
\mu_{0}\left(u, \chi_{10}, *\right)=\frac{1}{10}\left(-16 \gamma_{3}+328\right) \geq 0 ; \quad \mu_{0}\left(u, \chi_{12}, *\right)=\frac{1}{10}\left(4 \gamma_{4}+282\right) \geq 0 ; \\
\mu_{0}\left(u, \chi_{14}, *\right)=\frac{1}{10}\left(4 \gamma_{5}+338\right) \geq 0 .
\end{gathered}
$$

Clearly $\gamma_{1} \in\{-3,2,7\}, \gamma_{2} \in\{-9,1,11,21,31\}$ and $\gamma_{3} \in\{3+5 k \mid-4 \leq k \leq 3\}$. It follows that the only possible integer solutions for $\left(v_{2 a}, v_{2 b}, v_{13 a}\right)$ are $(0,-2,0,3),(0,-2,5,-2)$, $(1,3,-5,2)$ and $(1,3,0,-3)$. 
- $\chi\left(u^{5}\right)=3 \chi(2 a)-2 \chi(2 b)$ and $\chi\left(u^{2}\right)=\chi(5 a)$. Applying Proposition 5, we obtain:

$$
\begin{gathered}
\mu_{2}\left(u, \chi_{3}, *\right)=\frac{1}{10}\left(2 \gamma_{1}+6\right) \geq 0 ; \quad \mu_{0}\left(u, \chi_{3}, *\right)=\frac{1}{10}\left(-8 \gamma_{1}+16\right) \geq 0 ; \\
\mu_{1}\left(u, \chi_{8}, *\right)=\frac{1}{10}\left(\gamma_{2}+29\right) \geq 0 ; \quad \mu_{5}\left(u, \chi_{8}, *\right)=\frac{1}{10}\left(-4 \gamma_{2}+44\right) \geq 0 ; \\
\mu_{5}\left(u, \chi_{10}, *\right)=\frac{1}{10}\left(16 \gamma_{3}+352\right) \geq 0 ; \quad \mu_{0}\left(u, \chi_{10}, *\right)=\frac{1}{10}\left(-16 \gamma_{3}+248\right) \geq 0 ; \\
\mu_{0}\left(u, \chi_{14}, *\right)=\frac{1}{10}\left(4 \gamma_{5}+418\right) \geq 0 .
\end{gathered}
$$

Clearly $\gamma_{1} \in\{-3,2\} \gamma_{2} \in\{-29,-19,-9,1,11\}$ and $\gamma_{3} \in\{3+5 k \mid-5 \leq k \leq 2\}$. It follows that the only possible integer solutions for $\left(v_{2 a}, v_{2 b}, v_{13 a}\right)$ are $(-2,-2,0,5)$ and $(1,3,0,-3)$.

- $\chi\left(u^{5}\right)=-3 \chi(2 a)+4 \chi(2 b)$ and $\chi\left(u^{2}\right)=\chi(5 a)$. Applying Proposition 5, we obtain:

$$
\begin{aligned}
\mu_{5}\left(u, \chi_{3}, *\right) & =\frac{1}{10}\left(8 \gamma_{1}+8\right) \geq 0 ; \quad \mu_{1}\left(u, \chi_{3}, *\right)=\frac{1}{10}\left(-2 \gamma_{1}-2\right) \geq 0 ; \\
\mu_{0}\left(u, \chi_{8}, *\right) & =\frac{1}{10}\left(4 \gamma_{2}+40\right) \geq 0 ; \quad \mu_{2}\left(u, \chi_{8}, *\right)=\frac{1}{10}\left(-\gamma_{2}+25\right) \geq 0 ; \\
\mu_{5}\left(u, \chi_{10}, *\right) & =\frac{1}{10}\left(16 \gamma_{3}+256\right) \geq 0 ; \quad \mu_{0}\left(u, \chi_{10}, *\right)=\frac{1}{10}\left(-16 \gamma_{3}+344\right) \geq 0 ; \\
\mu_{0}\left(u, \chi_{14}, *\right) & =\frac{1}{10}\left(4 \gamma_{5}+322\right) \geq 0 ; \quad \mu_{5}\left(u, \chi_{14}, *\right)=\frac{1}{10}\left(-4 \gamma_{5}+388\right) \geq 0 .
\end{aligned}
$$

Clearly $\gamma_{1}=-1, \gamma_{2} \in\{-5,5,15,25\}$ and $\gamma_{3} \in\{4+5 k \mid-4 \leq k \leq 3\}$. It follows that there are no possible integer solutions for $\left(v_{2 a}, v_{2 b}, v_{13 a}\right)$ in this case.

Case $(v)$. Let $u \in V(\mathbb{Z} G)$ where $|u|=13$. By Proposition $2, v_{k x}=0$ for all

$$
\begin{array}{r}
k x \in\{2 a, 2 b, 3 a, 4 a, 4 b, 4 c, 5 a, 6 a, 8 a, 8 b, 8 c, 10 a, 12 a, 16 a, \\
16 b, 4 d, 4 e, 4 f, 4 g, 8 d, 8 e, 12 b, 12 c, 16 c, 16 d, 20 a, 20 b\} .
\end{array}
$$

Therefore, $u$ is rationally conjugated to some element $g \in G$ by Proposition 4 .

Case (vi). Let $u \in V(\mathbb{Z} G)$ where $|u|=15$. Using Propositions 2 and 3,

$$
v_{3 a}+v_{5 a}=1 .
$$

Applying Proposition 5, we obtain the following system of inequalities:

$$
\mu_{5}\left(u, \chi_{3}, *\right)=\frac{1}{15}(8 \gamma+62) \geq 0 ; \quad \mu_{0}\left(u, \chi_{3}, *\right)=\frac{1}{15}(-16 \gamma+56) \geq 0
$$

where $\gamma=\nu_{3 a}-v_{5 a}$. Clearly $\gamma=-4$ and there are no possible integer solutions for $\left(v_{3 a}, v_{5 a}\right)$.

Case (vii). Let $u \in V(\mathbb{Z} G)$ where $|u|=26$. Using Propositions 2 and 3,

$$
v_{2 a}+v_{2 b}+v_{13 a}=1 .
$$

Let $\gamma_{1}=3 v_{2 a}-v_{2 b}, \gamma_{2}=7 v_{2 a}-v_{2 b}$ and $\gamma_{3}=-20 v_{2 a}-4 v_{2 b}+v_{13 a}$. We shall now separately consider the following cases involving $\chi\left(u^{n}\right)$ for $n \in\{2,13\}$ :

- $\chi\left(u^{13}\right)=m_{1} \chi(2 a)+m_{2} \chi(2 b)$ and $\chi\left(u^{2}\right)=\chi(13 a)$ where $\left(m_{1}, m_{2}\right) \in\{(1,0)$, $(2,-1),(-1,2),(-2,3)\}$. Applying Proposition 5, we obtain:

$$
\mu_{13}\left(u, \chi_{3}, *\right)=\frac{1}{26}\left(48 \gamma_{1}+k_{1}\right) \geq 0 ; \quad \mu_{0}\left(u, \chi_{3}, *\right)=\frac{1}{26}\left(-48 \gamma_{1}+k_{2}\right) \geq 0
$$

where $\left(k_{1}, k_{2}\right)=(64,40)$ when $\left(m_{1}, m_{2}\right)=(1,0),\left(k_{1}, k_{2}\right)=(80,24)$ when $\left(m_{1}, m_{2}\right)=$ $(2,-1),\left(k_{1}, k_{2}\right)=(32,72)$ when $\left(m_{1}, m_{2}\right)=(-1,2)$ and $\left(k_{1}, k_{2}\right)=(16,80)$ when $\left(m_{1}, m_{2}\right)=(-2,3)$. It follows that there are no possible integer solutions for $\left(v_{2 a}, v_{2 b}, v_{13 a}\right)$ in all cases. 
- $\chi\left(u^{13}\right)=-3 \chi(2 a)+4 \chi(2 b)$ and $\chi\left(u^{2}\right)=\chi(13 a)$. Applying Proposition 5, we obtain:

$$
\begin{aligned}
\mu_{13}\left(u, \chi_{3}, *\right) & =\frac{1}{26}\left(48 \gamma_{1}\right) \geq 0 ; \quad \mu_{1}\left(u, \chi_{3}, *\right)=\frac{1}{26}\left(-4 \gamma_{1}\right) \geq 0 ; \\
\mu_{0}\left(u, \chi_{8}, *\right) & =\frac{1}{26}\left(24 \gamma_{2}+28\right) \geq 0 ; \quad \mu_{13}\left(u, \chi_{8}, *\right)=\frac{1}{26}\left(-24 \gamma_{2}+128\right) \geq 0 .
\end{aligned}
$$

It follows that there are no possible integer solutions for $\left(v_{2 a}, v_{2 b}, v_{13 a}\right)$.

- $\chi\left(u^{13}\right)=\chi(2 b)$ and $\chi\left(u^{2}\right)=\chi(13 a)$. Applying Proposition 5, we obtain:

$$
\begin{gathered}
\mu_{13}\left(u, \chi_{3}, *\right)=\frac{1}{26}\left(48 \gamma_{1}+48\right) \geq 0 ; \quad \mu_{0}\left(u, \chi_{3}, *\right)=\frac{1}{26}\left(-48 \gamma_{1}+56\right) \geq 0 ; \\
\mu_{0}\left(u, \chi_{8}, *\right)=\frac{1}{26}\left(24 \gamma_{2}+76\right) \geq 0 ; \quad \mu_{13}\left(u, \chi_{8}, *\right)=\frac{1}{26}\left(-24 \gamma_{2}+80\right) \geq 0 ; \\
\mu_{1}\left(u, \chi_{10}, *\right)=\frac{1}{26}\left(\gamma_{3}+303\right) \geq 0 .
\end{gathered}
$$

It follows that there are no possible integer solutions for $\left(v_{2 a}, v_{2 b}, v_{13 a}\right)$.

- $\chi\left(u^{13}\right)=3 \chi(2 a)-2 \chi(2 b)$ and $\chi\left(u^{2}\right)=\chi(13 a)$. Applying Proposition 5, we obtain:

$$
\begin{aligned}
& \mu_{2}\left(u, \chi_{3}, *\right)=\frac{1}{26}\left(4 \gamma_{1}+8\right) \geq 0 ; \quad \mu_{0}\left(u, \chi_{3}, *\right)=\frac{1}{26}\left(-48 \gamma_{1}+8\right) \geq 0 ; \\
& \mu_{0}\left(u, \chi_{8}, *\right)=\frac{1}{26}\left(24 \gamma_{2}+124\right) \geq 0 ; \quad \mu_{13}\left(u, \chi_{8}, *\right)=\frac{1}{26}\left(-24 \gamma_{2}+32\right) \geq 0 .
\end{aligned}
$$

It follows that there are no possible integer solutions for $\left(v_{2 a}, v_{2 b}, v_{13 a}\right)$.

Case (viii). Let $u \in V(\mathbb{Z} G)$ where $|u|=39$. Using Propositions 2 and 3,

$$
v_{3 a}+v_{13 a}=1
$$

Applying Proposition 5, we obtain the following system of inequalities:

$$
\begin{gathered}
\mu_{13}\left(u, \chi_{3}, *\right)=\frac{1}{39}\left(24 v_{3 a}+54\right) \geq 0 ; \quad \mu_{0}\left(u, \chi_{3}, *\right)=\frac{1}{39}\left(-48 v_{3 a}+48\right) \geq 0 ; \\
\mu_{1}\left(u, \chi_{3}, *\right)=\frac{1}{39}\left(-2 v_{3 a}+54\right) \geq 0 .
\end{gathered}
$$

Clearly $\nu_{3 a}=1$ and there are no possible integer solutions for $\left(\nu_{3 a}, v_{5 a}\right)$.

Case $(i x)$. Let $u \in V(\mathbb{Z} G)$ where $|u|=65$. Using Propositions 2 and 3,

$$
v_{5 a}+v_{13 a}=1
$$

Applying Proposition 5, we obtain the following system of inequalities:

$$
\mu_{0}\left(u, \chi_{3}, *\right)=\frac{1}{65}\left(96 v_{5 a}+60\right) \geq 0 ; \quad \mu_{13}\left(u, \chi_{3}, *\right)=\frac{1}{65}\left(-24 v_{5 a}+50\right) \geq 0 ;
$$

Clearly there are no possible integer solutions for $\left(v_{5 a}, v_{13 a}\right)$.

We shall now consider the prime graph of $G={ }^{2} F_{4}(2)$. Again, $G$ contains elements of order 6 and 10. Therefore [2,3] and [2,5] are adjacent in $\pi(G)$ and consequently adjacent in $\pi(V(\mathbb{Z} G))$. Clearly $\pi(G)=\pi(V(\mathbb{Z} G))$, since there are no torsion units of order 15,26 , 39 and 65 in $V(\mathbb{Z} G)$. This completes the proof.

Open Access This article is distributed under the terms of the Creative Commons Attribution 4.0 International License (http://creativecommons.org/licenses/by/4.0/), which permits unrestricted use, distribution, and reproduction in any medium, provided you give appropriate credit to the original author(s) and the source, provide a link to the Creative Commons license, and indicate if changes were made. 


\section{References}

1. Zassenhaus, H.: On the torsion units of finite group rings, Studies in mathematics (in honor of A. Almeida Costa) (Portuguese), pp. 119-126. Instituto de Alta Cultura, Lisbon (1974)

2. Roggenkamp, K., Scott, L.: Isomorphisms of p-adic group rings. Ann. Math. (2) 126(3), 593-647 (1987). doi: $10.2307 / 1971362$

3. Weiss, A.: Rigidity of $p$-adic p-torsion. Ann. Math. (2) 127(2), 317-332 (1988). doi:10.2307/2007056

4. Caicedo, M., Margolis, L., del Río, Á.: Zassenhaus conjecture for cyclic-by-abelian groups. J. Lond. Math. Soc. (2) 88(1), 65-78 (2013)

5. Luthar, I.S., Passi, I.B.S.: Zassenhaus conjecture for $A_{5}$. Proc. Indian Acad. Sci. Math. Sci. 99(1), 1-5 (1989)

6. Höfert, C., Kimmerle, W.: On torsion units of integral group rings of groups of small order, Groups, rings and group rings, Lect. Notes Pure Appl. Math., vol. 248, pp. 243-252. Boca Raton: Chapman \& Hall/CRC (2006)

7. Bovdi, V., Hertweck, M.: Zassenhaus conjecture for central extensions of $S_{5}$. J. Group Theory 11(1), 63-74 (2008)

8. Bovdi, V., Höfert, C., Kimmerle, W.: On the first Zassenhaus conjecture for integral group rings. Publ. Math. Debrecen 65(3-4), 291-303 (2004)

9. Luthar, I.S., Trama, Poonam: Zassenhaus conjecture for $S_{5}$. Commun. Algebra 19(8), 2353-2362 (1991)

10. Salim, M.: Torsion units in the integral group ring of the alternating group of degree 6 . Commun. Algebra 35(12), 4198-4204 (2007). doi:10.1080/00927870701545069

11. Hertweck, M.: Zassenhaus conjecture for $A_{6}$. Proc. Indian Acad. Sci. Math. Sci. 118(2), 189-195 (2008)

12. Salim, M.: Kimmerle's conjecture for integral group rings of some alternating groups. Acta Math. Acad. Paedagog. Nyházi. (N.S.) 27(1), 9-22 (2011)

13. Salim, M.: The prime graph conjecture for integral group rings of some alternating groups. Int. J. Group Theory 2(1), 175-185 (2013)

14. Hertweck, M.: Partial augmentations and Brauer character values of torsion units in group rings. arXiv:math/0612429 (2007)

15. Gildea, J.: Zassenhaus conjecture for integral group ring of simple linear groups. J. Algebra Appl. 12(6), 1350016 (2013). 10

16. Bachle, A., Margolis, L.: Rational conjugacy of torsion units in integral group rings of non-solvable groups. arXiv:1305.7419

17. Hertweck, M., Höfert, C., Kimmerle, W.: Finite groups of units and their composition factors in the integral group rings of the group PSL $(2, q)$. J. Group Theory 12(6), 873-882 (2009)

18. Margolis, L.: A Sylow theorem for the integral group ring of $P \operatorname{SL}(2, q)$. arXiv: 1408.6075

19. Mini-Workshop, Arithmetik von Gruppenringen, Oberwolfach Rep. 4 (2007), no. 4, 3209-3239, Abstracts from the mini-workshop held November 25-December 1, 2007, Organized by E. Jespers, Z. Marciniak, G. Nebe and W. Kimmerle, Oberwolfach Reports. vol. 4, no. 4

20. Kimmerle, W.: On the prime graph of the unit group of integral group rings of finite groups, Groups, rings and algebras, Contemp. Math., vol. 420, pp. 215-228. Amer. Math. Soc., Providence (2006)

21. Bovdi, V., Grishkov, A., Konovalov, A.: Kimmerle conjecture for the Held and O'Nan sporadic simple groups. Sci. Math. Jpn. 69(3), 353-361 (2009)

22. Bovdi, V., Jespers, E., Konovalov, A.B.: Torsion units in integral group rings of Janko simple groups. Math. Comput. 80(273), 593-615 (2011). doi:10.1090/S0025-5718-2010-02376-2

23. Bovdi, V., Konovalov, A.B.: Integral group ring of the first Mathieu simple group, Groups St. Andrews 2005. vol. 1, London Math. Soc. Lecture Note Ser., vol. 339, pp. 237-245. Cambridge: Cambridge Univ. Press (2007)

24. Bovdi, V., Konovalov, A.B.: Integral group ring of the Mathieu simple group $M_{23}$. Comm. Algebra 36(7), 2670-2680 (2008)

25. Bovdi, V., Konovalov, A.B.: Integral group ring of Rudvalis simple group, Ukraïn. Mat. Zh. 61(1), 3-13 (2009) (English, with English and Ukrainian summaries); English transl., Ukrainian Math. J. 61(1), 1-13 (2009)

26. Bovdi, V., Konovalov, A.B.: Torsion units in integral group ring of Higman-Sims simple group. Stud. Sci. Math. Hungar. 47(1), 1-11 (2010)

27. Bovdi, V., Konovalov, A.B.: Integral group ring of the McLaughlin simple group. Algebra Discrete Math. 2, 43-53 (2007)

28. Bovdi, V., Konovalov, A.B.: Integral group ring of the Mathieu simple group $M_{24}$. J. Algebra Appl. 11(1), 1250016 (2012). 10

29. Bovdi, V., Konovalov, A.B., Linton, S.: Torsion units in integral group ring of the Mathieu simple group $M_{22}$. LMS J. Comput. Math. 11, 28-39 (2008) 
30. Bovdi, V., Konovalov, A.B., Linton, S.: Torsion units in integral group rings of Conway simple groups. Int. J. Algebra Comput. 21(4), 615-634 (2011)

31. Bovdi, V.A., Konovalov, A.B., Marcos, E.N.: Integral group ring of the Suzuki sporadic simple group. Publ. Math. Debrecen 72(3-4), 487-503 (2008)

32. Bovdi, V., Konovalov, A.B., Siciliano, S.: Integral group ring of the Mathieu simple group $M_{12}$. Rend. Circ. Mat. Palermo (2) 56(1), 125-136 (2007)

33. Rosa, A.: Torsion units of integral group ring of the simple group $S_{4}(4)$. Miskolc Math. Notes 16(1), 443-452 (2015)

34. Steinberg, R.: Variations on a theme of Chevalley. Pacif. J. Math. 9, 875-891 (1959)

35. Ree, R.: A family of simple groups associated with the simple Lie algebra of type $\left(F_{4}\right)$. Bull. Am. Math. Soc. 67, 115-116 (1961)

36. Tits, J.: Algebraic and abstract simple groups. Ann. Math. (2) 80, 313-329 (1964)

37. Hertweck, M.: On the torsion units of some integral group rings. Algebra Colloq. 13(2), 329-348 (2006)

38. Artamonov, V.A., Bovdi, A.A.: Integral group rings: groups of invertible elements and classical $K$-theory, Algebra. Topology. Geometry, vol. 27 (Russian), Itogi Nauki i Tekhniki, Akad. Nauk SSSR Vsesoyuz. Inst. Nauchn. i Tekhn. Inform., Moscow, 1989, pp. 3-43, 232 (Russian). Translated in J. Soviet Math. 57(2), 2931-2958 (1991)

39. Cohn, J.A., Livingstone, D.: On the structure of group algebras. I. Can. J. Math. 17, 583-593 (1965)

40. Bovdi, V., Konovalov, A., Rossmanith, R., Schneider, C.: LAGUNA-Lie AlGebras and UNits of group Algebras, Version 3.5.0; 2009 (andrews.ac.uk/alexk/laguna.htm http://www.cs.st)

41. The GAP Group, GAP- Groups, Algorithms and Programming, version 4.4 2006. (http://www.gapsystem. org) 